\title{
A review of graphene oxide, graphene buckypaper, and polymer/graphene composites: Properties and fabrication techniques
}

Journal of Plastic Film \& Sheeting 2016, Vol. 32(4) 336-379

(C) The Author(s) 2015 Reprints and permissions: sagepub.co.uk/journalsPermissions.nav DOI: $|0.1| 77 / 87560879|56| 46 \mid 2$ jpf.sagepub.com

@SAGE

\author{
Zaheen U Khan ',2, Ayesha Kausar', \\ Hidayat Ullah ${ }^{2}$, Amin Badshah $^{3}$ \\ and Wasid $U$ Khan ${ }^{4}$
}

\begin{abstract}
In this critical review, the preparation methods, unique properties, and graphene oxide structure as well as graphene have been interpreted. Various aspects for the graphene oxide reactivity were also discussed. Graphene oxide, usually obtained by exfoliating graphite, provides a way towards flexible, low-cost, bulk production paper-like materials known as buckypapers. Recently, graphene oxide and graphene buckypaper have been used as fillers for preparing composites. The buckypapers are freestanding thin porous network of carbon fillers bound by van der Waals interactions. Several techniques have been used to fabricate graphene oxide and graphene-based paper-like materials such as vacuum filtration method; spin coating technique, resin infiltration technique, evaporation-induced, and self-assembly technique. Among these, the most suitable technique is Langmuir-Blodgett
\end{abstract}

\footnotetext{
'Nanosciences and Catalysis Division, National Center For Physics, Quaid-i-Azam University Campus, Islamabad, Pakistan

${ }^{2}$ Institute of Chemical Sciences, Gomal University, Dera Ismail Khan, Pakistan

${ }^{3}$ Department of Chemistry, Quaid-i-Azam University, Islamabad, Pakistan

${ }^{4}$ Analytical Chemistry Department, Institute of Chemistry, São Paulo State University, Araraquara-SP, Brazil

Corresponding author:

Ayesha Kausar, Nanosciences and Catalysis Division, National Center For Physics, Quaid-i-Azam University Campus, Islamabad, Pakistan.

Email: asheesgreat@yahoo.com
} 
assembly, where the graphene oxide paper formed is usually highly uniform. Free standing polymer/graphene oxide and polymer/graphene paper materials show remarkable mechanical stiffness and excellent flexibility combined with outstanding electrical conductivity. Incorporating polymer into graphene oxide and graphene buckypaper may modify the polymer and filler properties producing high performance materials. The buckypaper made from functionalized filler may form networks that facilitate penetrating matrix chains and result in composites with improved mechanical performance. Due to their outstanding physical, thermal, and electrical properties, these papers have promising applications such as; body armour, aerospace structure improvement, armoured vehicles, flexible energy storage devices, sensors, memory devices, transparent conductors, and photovoltaics.

\section{Keywords}

Buckypaper, graphene oxide, graphene, Langmuir-Blodgett assembly, freestanding, polymer/graphene paper

\section{Introduction}

The name 'Carbon' is derived from Carbo which is a Latin word meaning charcoal. This element forms $\mathrm{sp}, \mathrm{sp}^{2}$ and $\mathrm{sp}^{3}$ networks due to exceptional electronic structure and has many stable allotropes. In carbon allotropes, graphite is a common allotropic form, which has been known since ancient times together with diamond. Graphite is an abundant natural mineral consisting of $\mathrm{sp}^{2}$ hybridized carbon atomic layers which are stacked together by van der Waals interactions. The single carbon atomic layer of graphite may compactly arrange into a two dimensional (2D) honeycomb lattice known as graphene. In 1994, Boehm et al. ${ }^{1}$ introduced this name. With respect to electrical conductivity and thermal properties, graphite displays significant anisotropic behavior. In the direction perpendicular to the graphene layers, graphite is a poor conductor due to the weak van der Waals forces between them. It exhibits high conductivity in the direction parallel to the graphene layers due to in-plane metallic character. ${ }^{2}$ The spacing between the graphite interlayer is $0.34 \mathrm{~nm}$ which is not sufficient for intercalating inorganic species or organic molecules or ions. However, to expand graphite's interplanar spacing from $0.34 \mathrm{~nm}$ to greater values, several strategies have been adopted. In some cases, the interlayer spacing reached more than $1 \mathrm{~nm}$ and it depends upon the guest molecule or ion size. Since the first graphite intercalation with potassium many chemical species have been tried and these compounds are known as graphite intercalation compounds (GICs). The inserted species between the graphene layers are stabilized through either polar or ionic forces without affecting the graphene structure. Not only the alkali metals 
such as potassium, lithium, and sodium have been intercalated among graphene layers but also halogens, bisulfate or nitrate formed graphite intercalation compounds. In some cases, the intercalation of guest species into graphene layers may happen via chemical bonding through chemical grafting reactions inside the graphite interplanar spaces. Due to these reactions, the $\mathrm{sp}^{2}$-hybridized carbon atoms change to $\mathrm{sp}^{3}$ which modify graphene structural planes. An example of this method is the insertion of oxidizing agent and strong acid that generates oxygen functionalities at the edges and on the surfaces of graphene layers. Due to this strong oxidative action, anionic groups mostly carboxylates, epoxy, and hydroxylate groups are formed on the graphitic layers. The interplanar distance between graphene layers increases by out of planar $\mathrm{C}-\mathrm{O}$ covalent bonding from $0.35 \mathrm{~nm}$ in graphite to $\sim 0.68 \mathrm{~nm}$ in graphene oxide (GO). Stankovich et al. ${ }^{3}$ reported that synthesizing graphene and graphene oxide is very attractive for many reasons such as:

- graphite is an inexpensive and ubiquitous resource and used as a raw material for their preparation.

- due to efficient exfoliation, the monolayer graphene and graphene oxide yield is very high $(>80 \%)$, thus a small mass transforms into atomically thin film with large surface area.

- the whole process is conducted in solution media allowing paper like materials fabrication or simple thin film deposition.

- chemical methods simplify integrating graphene and graphene oxide into composites with ceramic or polymeric hosts. ${ }^{4}$

Several techniques have been used to fabricate graphene and graphene oxide paper-like materials or thin film deposition such as; vacuum filtration assembly, evaporation induced self-assembly, spin coating, drop-casting, Langmuir-Blodgett (L-B), spraying, dip coating and Langmuir-Schaefer methods. ${ }^{5}$ Due to processing advantages and extra-ordinary properties such as; optical, tunable electrical properties and thermal stability, graphene and graphene oxide are very attractive for bendable and flexible thin film optoelectronics where different device components are formed on paper-like or plastic platforms. Analogous to carbon nanotubes network structures, the graphene and graphene oxide sheets can be assembled into multi layered network structures which can be seen as polycrystalline thin films where the single crystal are the individual graphene and graphene oxide. ${ }^{6}$ In past years, numerous reports have been appeared, revealing the unique properties of graphene and graphene oxide based thin film and paper-like materials providing prospects for their use as chemical/biological sensors, transparent conductors, electrodes, thin film transistors, ultracapacitors, photodetectors, field emitters and nonvolatile memory devices. ${ }^{5}$ In this critical review, the graphene and graphene oxide chemistry including history, synthesis, structure 
and properties will be summarized. Subsequently, key demonstration of graphene and graphene oxide papers is highlighted. The consideration emphasizing the requirement for an enhanced understanding of the structureproperty relationship of the paper-like materials is presented. Here, the research relies on basics for the production of flexible high performance energy storage devices. The GO paper, fabrication techniques, polymer/graphene, and polymer/GO composite papers including their properties will be presented.

\section{Graphene}

\section{History of graphene}

During recent decades, graphene attracted much attention in the field of science and technology. To understand the graphene history, first of all some terms are important to discuss such as graphite oxide (GO), exfoliation of graphite i.e. graphene oxide, graphite intercalation compounds and graphene. Initially $\mathrm{GO}$ was synthesized from the exfoliation of graphite using $\mathrm{HNO}_{3}$ and $\mathrm{H}_{2} \mathrm{SO}_{4}$. In 1859, a British scientist, Brodie ${ }^{7}$ modified the Schafhaeut method by using some oxidants like $\mathrm{KClO}_{3}$ along with $\mathrm{H}_{2} \mathrm{SO}_{4}$ and $\mathrm{HNO}_{3}$. As a result, he observed graphite layer intercalation and GO surface chemical oxidation. The GO surface chemical modification caused the lamellar stacking by decreasing the interplanar forces. Hence, these oxidized GO layers can be easily exfoliated under thermal, ultrasonic, and other energetic conditions. In order to synthesize graphene, it has been very essential to prepare GO. Hummer and Offman, ${ }^{8}$ in 1858, recommended a different GO oxidation process by reacting graphite with potassium permanganate $\left(\mathrm{KMnO}_{4}\right)$ and concentrated sulfuric acid. The most common process for GO production is the modified Hummer method. In 1962, Boehm et al. ${ }^{9}$ made the first observation about graphene-like structures. They synthesized reduced GO by reducing a GO dispersion in alkaline solution. They concluded that this method produced thin lamella carbon with very low oxygen and hydrogen content. The so called 'graphite oxide soot' derived from combusting graphite oxide at high heating rate was investigated. Electron microscopy revealed that this soot consisted of thin platelet structure of multi-layer carbon hexagonal sheets (nearly $12.6 \AA$ equal to 2-3 layers) is known as graphene nanoplatelets. In the meanwhile, graphite and graphite intercalation compounds received much attention, as they presented a realistic route for ion storage. Hence, Boehm et al. ${ }^{1}$ in 1994 introduced the new terminology for graphite intercalation compound termed as graphene, which is a single carbon layer in graphite structure. Blakely et al., ${ }^{10}$ in 1970, found that graphite monolayers were produced by isolating carbon on a nickel surface. The carbon was dissolved when exposed to high temperature and the phase was found to 
separate to form single or multiple carbon layers on a metal surface. In describing graphene, until 1995, several different terms were used such as graphite layers, carbon layers or carbon sheets. After that IUPAC decided on a definition for graphene which is "A single carbon layer of the graphite structure, describing its nature by analogy to a polycyclic aromatic hydrocarbon of quasi infinite size." The word graphene should be used only when structural relations, reactions or other characteristics of specific layers was discussed. ${ }^{11}$ In 2004, Novoselov et al. ${ }^{12}$ presented their conclusions on the structure of graphene. These results were a key milestone in the history of graphene, emerging as a new field of science. They used an easy and operative mechanical method for the exfoliation and extraction of thin graphene layers from graphite crystals using a Scotch tape method. Then the graphene layers were moved to a silicon substrate. These graphene fragments made up of single layers were identified by Atomic Force Microscopy (AFM). Graphene was discovered earlier than 2004, but at this time scientists needed to isolate individual graphene sheets in order to find, characterize and confirm its distinctive two-dimensional properties (Figure 1). For this work, they were awarded with the Nobel Prize in physics in 2010.

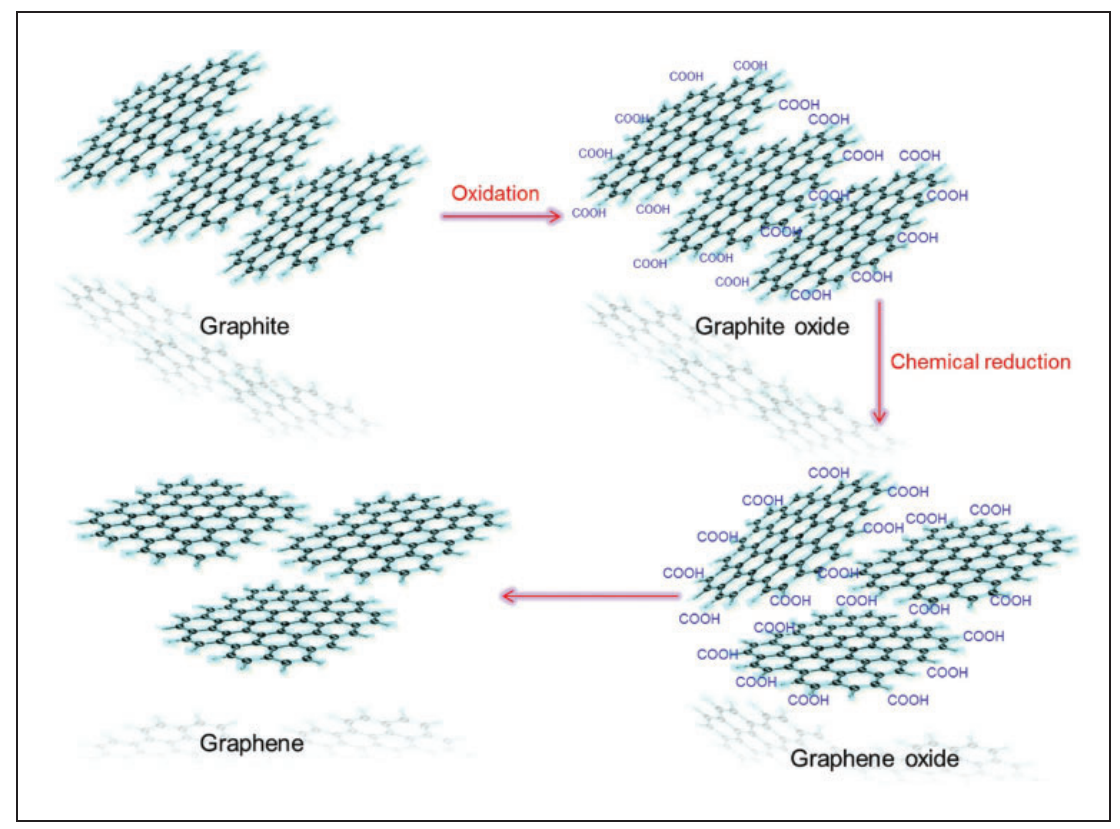

Figure I. Schematic showing how graphite is transformed to graphite oxide, then to graphene oxide, and finally graphene. 


\section{Properties of graphene}

At present, graphene is an exciting material and has been widely used because of its low percolation threshold. The low percolation threshold is related to other significant properties such as barrier resistance, electrical conductivity, electrooptics, abrasion resistance, stiffness, and fire retardancy at low filler loading content. ${ }^{13}$ Particularly, it shows conductivity behavior and unusual fractional quantum hall effect, due to which it has been used in many technological fields such as nanocomposites, sensors, batteries, and super-capacitors. ${ }^{14}$ Graphene is rapidly gaining attention from a broad spectrum of research areas due to its remarkable properties including high mechanical strength, high carrier mobility $\left(200,000 \mathrm{~cm}^{2} /(\mathrm{V} . \mathrm{s})\right)$, excellent electrical conductivity, and excellent thermal properties. Within ideal graphene layers, the $\mathrm{sp}^{2}$ hybridized carbon atoms are highly well-organized in shuttling and storing electrons. Hence, the graphene and graphene-based materials hold a great potential in applications (energy storage devices, field emission displays (FEDs) and photo-catalysis) where fast electron transfer is required. Figure 2 shows AFM and TEM images of

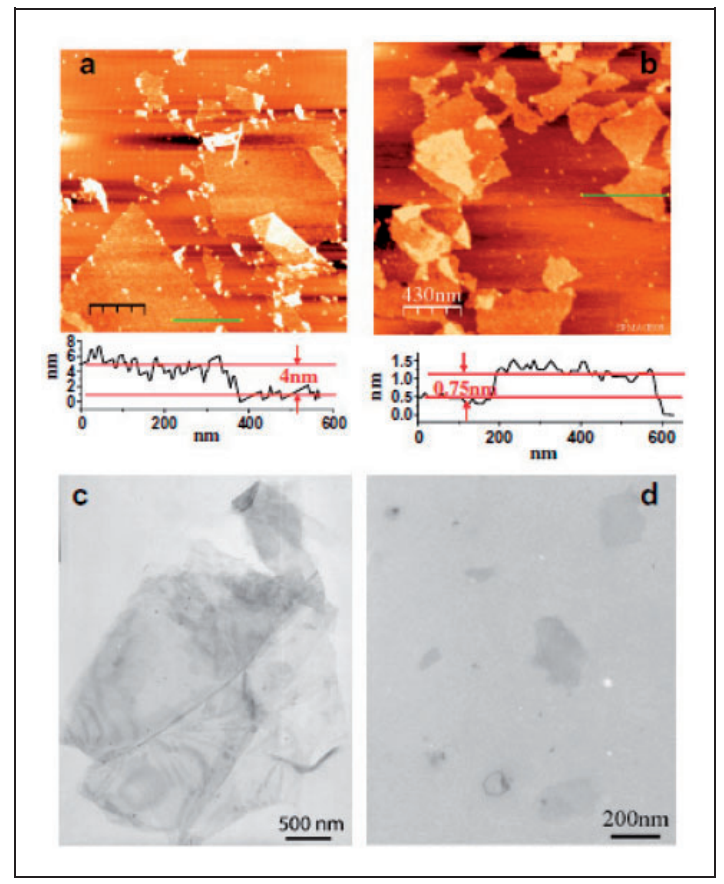

Figure 2. Imaging characterizations of graphene and its polymer composites. (a) AFM images and graphene/polymer composite height profile. (b) AFM image and thermally annealed graphene height profile. (c) Thermally annealed graphene TEM image directly exfoliated from graphite and (d) Graphene TEM image prepared by oxidation-reduction method. ${ }^{15}$ 
graphene and graphene/polymer composites. ${ }^{15}$ The graphene lattice is twodimensional and is based on honeycomb structure with hexagonal geometry of quasi infinite size. Furthermore, graphene's remarkable properties depend on the two-dimensional crystal lattice. The carbon atoms within the lattice are $\mathrm{sp}^{2}$ hybridized and therefore each carbon is interconnected to its three neighbors through $\sigma$ bonds. The bond angles are $120^{\circ}$ and the bond length is $1.42 \AA$ (Figure 3).

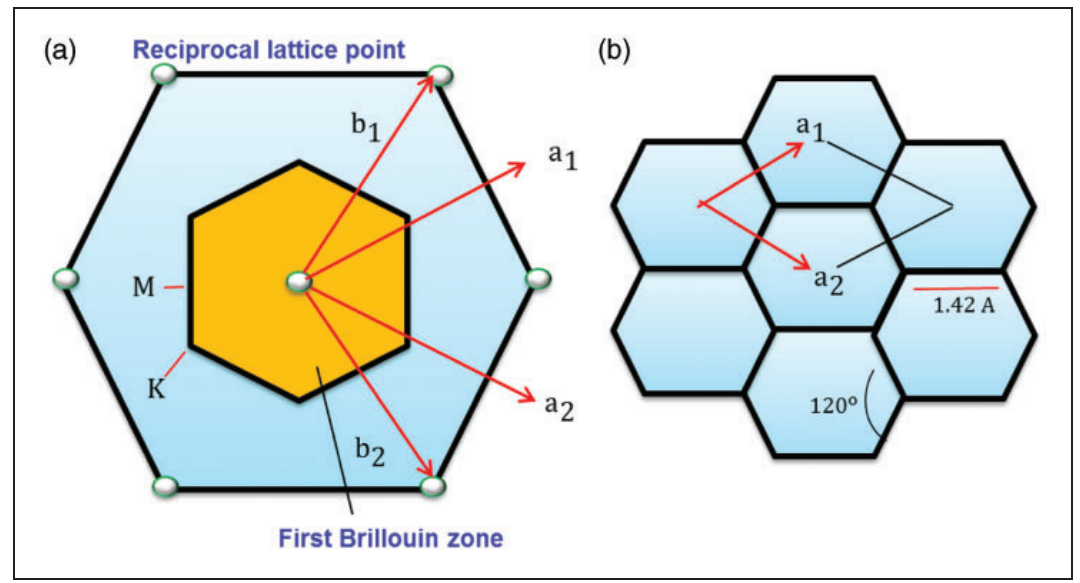

Figure 3. (a) Corresponding reciprocal lattice with unit cell vectors $b_{1}$ and $b_{2}$; and (b) lattice structure of graphene with lattice unit vectors $a_{1}$ and $a_{2}$.

The $\sigma$ bonds are responsible for the graphene lattice toughness. According to Pauli Exclusion Principle, these bonds formed a deep valence band because of filled shell. The $\mathrm{p}_{\mathrm{z}}$ orbital are covalently bound in the form of $\pi$ bonds with the neighboring carbon atom. It provides one electron each and the subsequent $\pi$ bonds are only half filled. ${ }^{16}$ In 1946 , Wallace ${ }^{17}$ described the first graphene band structure and emphasized the semi-metallic nature of graphene. The overall band structure of graphene was designated as a semiconductor having a zero band gap or as a metal with disappearing Fermi surface.

\section{Graphene synthesis}

Micromechanical cleavage. Micromechanical cleavage comprises continually peeling the graphite layers. To peel off the graphite layers from the surface, an adhesive tape is used and successive peeling leads to a single graphite layer on the silicon dioxide $\left(\mathrm{SiO}_{2}\right)$ surface. In this process, the size reported for single layer is up to $10 \mu \mathrm{m}$, while the size of thicker multilayer films is about $100 \mu \mathrm{m} .{ }^{18}$ These sheets are visible under an optical microscope due to optical 
interference contrast. Although this method is very crude, it has proven to be very dependable and has been used for several research investigations. However, the yield obtained by this method is very low i.e. per $\mathrm{mm}^{2}$ area of substrate few graphene monolayers has been achieved. Micromechanical exfoliation method can be used to produce graphene with high quality that is isolated electrically for fundamental transport physics studies and many other properties. Yet this method is not accessible to large area. Typically, it produces $10-100 \mu \mathrm{m}$ graphene particles. Ruoff ${ }^{19}$ invented pillar approach to achieve patterned graphene from patterned graphite by micromechanical exfoliation. Many modifications occurred during the exfoliation technique to enhance the exfoliated single layer graphite yield. Figure 4 shows removal printing graphene layers from graphite crystal using polydimethyl siloxane (PDMS) stamping. As compared to tape exfoliation, this method also follows similar principles. However, it offers the improved and controlled sheet placement. Moreover, the single layer graphene yield and size still remains a bottleneck in this process. ${ }^{20}$

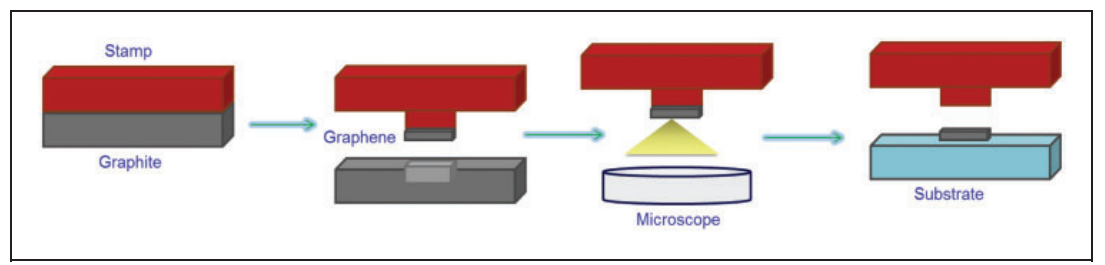

Figure 4. Graphene via PDMS stamp printing.

Chemical vapor deposition method for graphene synthesis. In recent years, growing graphene layers on the surface of transition metals has attracted great interest. The basic principle of this process is the thermal decomposition of hydrocarbons on the surface of transition metals. Due to precipitation of graphite from a hydrocarbon species on Ni surface, graphene growth occurs. Moreover, the carbon atoms continuously form a solid solution on $\mathrm{Ni}$ surface resulting in ultrathin film of graphene ( 1 to $\sim 10$ layers). ${ }^{21}$ To grow carbon nanotube, chemical vapor deposition (CVD) has been very successful over the years in producing tubes with high yield and high quality (Figure 5). Similar to carbon nanotube $(\mathrm{CNT})$, this technique is also used to produce single layer graphene. The process starts with a flat transition metals substrate surface and a proper carbon precursor like methane and ethylene. The transition metal substrate acts as a catalyst placed in a heated furnace and then attached with gas delivery system. The carbon precursor molecules from the liquid phase at elevated temperature or from gas phase at low pressure will be brought 


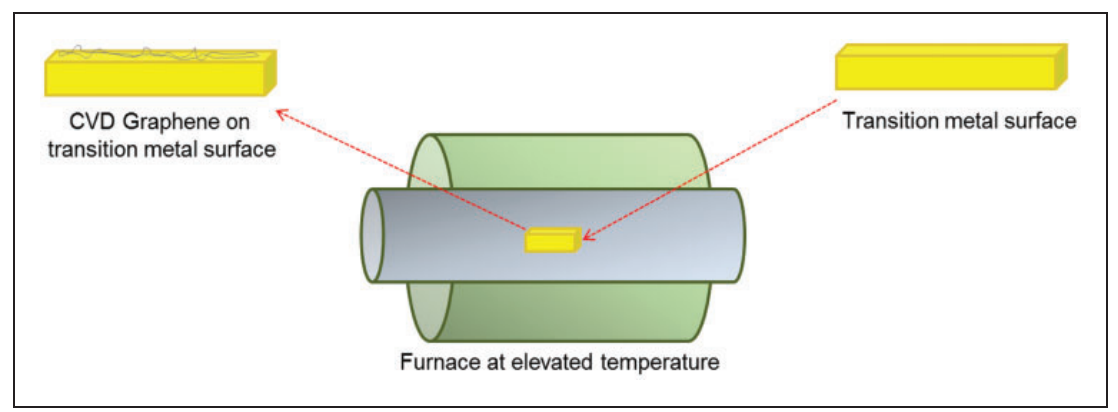

Figure 5. Graphene synthesion via CVD method.

into interaction with a metal substrate surface. The precursor molecules with the surface are cracked to form carbon atoms and gas phase species, upon contacting. The carbon precursor leaves the carbon atoms which are free of functionalities and attach to the surface. The carbon atoms diffuse on the substrate surface and graphene layers are formed. The conditions have been controlled carefully in order to get a single layer graphene with lowest energy. ${ }^{22}$ Initial observation of graphene growth by CVD was made on the surface of Ir (III) or Ru (III) using an ethylene precursor under ultra-high vacuum (UHV) conditions. The graphene layers about $200 \mu \mathrm{m}$ have been observed to nucleate by segregation of carbon on the surface upon cooling. Although this method allows the growing single layer graphene, the main issue is that the first graphene layer adheres to the metal substrate. The second layers are decoupled and recover the electronic structure of graphene.

Solution-based exfoliation technique. Using a metal substrate is a problem with the CVD technique which often necessitates transferring graphene onto insulating substrates. Moreover, the mechanical exfoliation does not give high monolayer yields. Therefore, the solution-based technique is used to solve the above problems and to separate the graphite layers to produce graphene suspension. The principle is based on weakening the van der Waals forces between the graphite layers either by functionalization or by intercalation of individual layers. This technique affords the chance of high yield production and offers high versatility in terms of being compatible to chemical functionalization. Hence, it is promising for new applications. The starting materials used to obtain the colloidal dispersion of graphene sheets are expandable graphite or graphite intercalation compounds (GIC). Ideally, this technique provides high quality single layer graphene sheets. The graphite powder was dispersed in organic solvent such as N-methylpyrrolidine (NMP) resulting in 
colloidal suspension of graphene sheets with a lateral size of a few hundred $\mathrm{nm}$ and very low yields. ${ }^{23}$ Electrical resistivity measurement had shown that the electrical conductivity of these sheets is $6500 \mathrm{Sm}^{-1}$ at $42 \%$ optical transparency. A milder dissolution way to obtain the large size graphene sheets has also been reported, where the staring materials is natural graphite. This was obtained by mechanically stirring a natural graphite intercalation compound i.e. ternary potassium salt $\left(\mathrm{K}(\mathrm{THF})_{\mathrm{x}} \mathrm{C}_{24}\right)$ in $\mathrm{NMP} .{ }^{24}$ Another method to obtain the separated graphene sheets was first to intercalate graphite with oleum and then expand with tetrabutyl ammonium hydroxide (TBA) as shown in Figure $6 .^{25}$

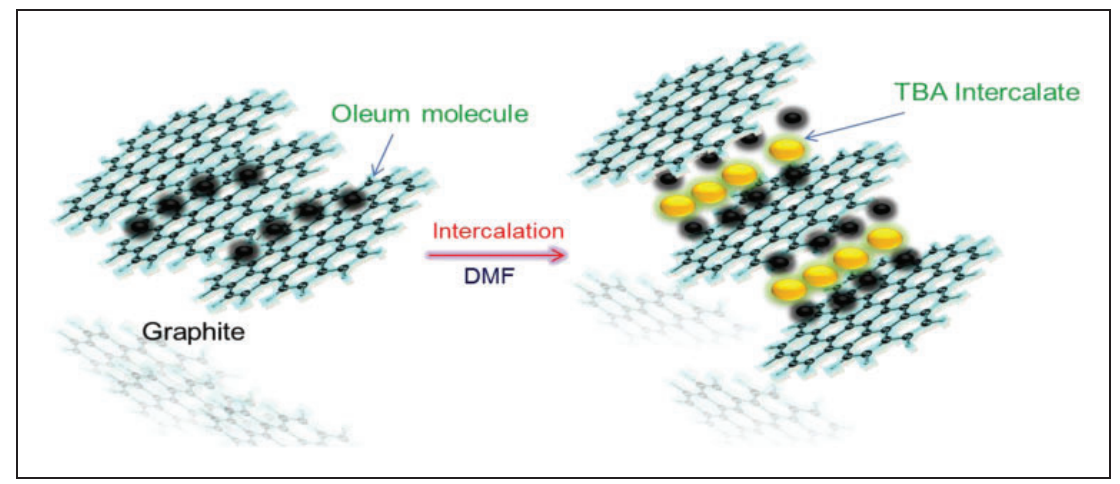

Figure 6. Graphite exfoliation intercalated with sulfuric acid followed by TBA insertion.

There have been various other approaches to exfoliate graphite in liquids in order to obtain a stable colloidal suspension of single layer graphene sheets. ${ }^{26}$ However, one of the most up scalable, widely studied, most promising, and low-cost synthetic method is the reduction of oxidized graphite layers with controllable density.

\section{Graphene oxide}

During the last few years, chemically modified graphene (CMG) has attracted great interest in the perspective of several applications such as sensors, energy related materials, polymer composites, field effect transistors (FET), paper-like materials, and biomedical relevance due to remarkable mechanical, thermal and electrical, properties. ${ }^{27}$ Chemically modified graphene oxide has been a favorable route to obtain mass produced CMG platelets. Similar to 
graphite, graphite oxide also has a layered structure, but in graphite oxide the carbon planes are highly decorated by oxygen containing functional groups. These functional groups not only make the layers hydrophilic but they also expand the interlayer distance. Under moderate ultrasonication, these oxidized layers are exfoliated in water resulting in the exfoliated sheets with one or few carbon layers like graphene. These sheets are also called graphene oxide (GO). ${ }^{28}$ Graphene oxide consists of pseudo-two dimensional carbon layers usually generated from graphite oxide. The primary precursor for synthesizing graphene oxide is graphite flakes which are first oxidized to graphite oxide. ${ }^{29}$ Graphene oxide contains reactive oxygen with functional groups such as carboxylic, hydroxyl, and epoxy groups which render it the best precursor in above mentioned applications. The epoxy and hydroxyl groups are attached on the GO basal plane, while the carboxylic groups are present on the edges of graphene oxide. However, due to these functional groups, GO is strongly hydrophilic in nature and disperse easily in water and intercalation of water molecules readily occur between the GO sheets. Depending upon the relative humidity within the stacked GO sheets, the interlayer spacing between GO sheets varies significantly from 0.6 to $1.2 \mathrm{~nm} .{ }^{30}$ Consequently, the interaction between GO sheets is weakened, while the inter-sheet spacing is increased which in turn enables the exfoliation of GO sheets. Mechanical stirring, thermal shock, ultra-sonication in water, or polar solvents are used to exfoliate GO into two dimensional individual nanosheets. ${ }^{31}$ However, many studies claimed that too much ultrasonication could decrease the lateral dimension of GO sheets. The resulting individual $\mathrm{GO}$ sheets are mostly single or few layer sheets that readily disperse in water to make a stable colloidal GO suspension. The GO suspension stability is assumed to be from negative electrostatic repulsion due to ionization of phenolic hydroxyl groups and carboxylic groups. ${ }^{32}$ As a result of the introduction of the oxygen functional groups on the carbon basal planes, the thickness of single-layer GO sheets has been reported approximately to be $1-1.4 \mathrm{~nm}$. In other words, the individual GO sheet thickness is approximately three times greater than an ideal single graphene layer ${ }^{33}$ Figure 7 shows GO sheet AFM and TEM images. Indeed, the graphite oxide exfoliation into individual GO sheet can also occur in polar organic solvents such as N-methylpyrolidine (NMP), ethylene glycol (EG) and N,N-dimethylformamide (DMF). It forms a non-aqueous colloidal suspension that is analogous to aqueous $\mathrm{GO}$ colloidal suspension. ${ }^{34}$ Generally, the GO sheets concentration, dispersed in water, is up to $3 \mathrm{mg} / \mathrm{ml}$. The aqueous GO colloidal suspension offers an appropriate setting to apply an electrochemical method to converting GO into electrochemically reduced graphene oxide (ERGO). However, the properties of ERGO are different from pristine graphene because of various residual oxygen containing functional groups on the carbon basal plane. ${ }^{35}$ 


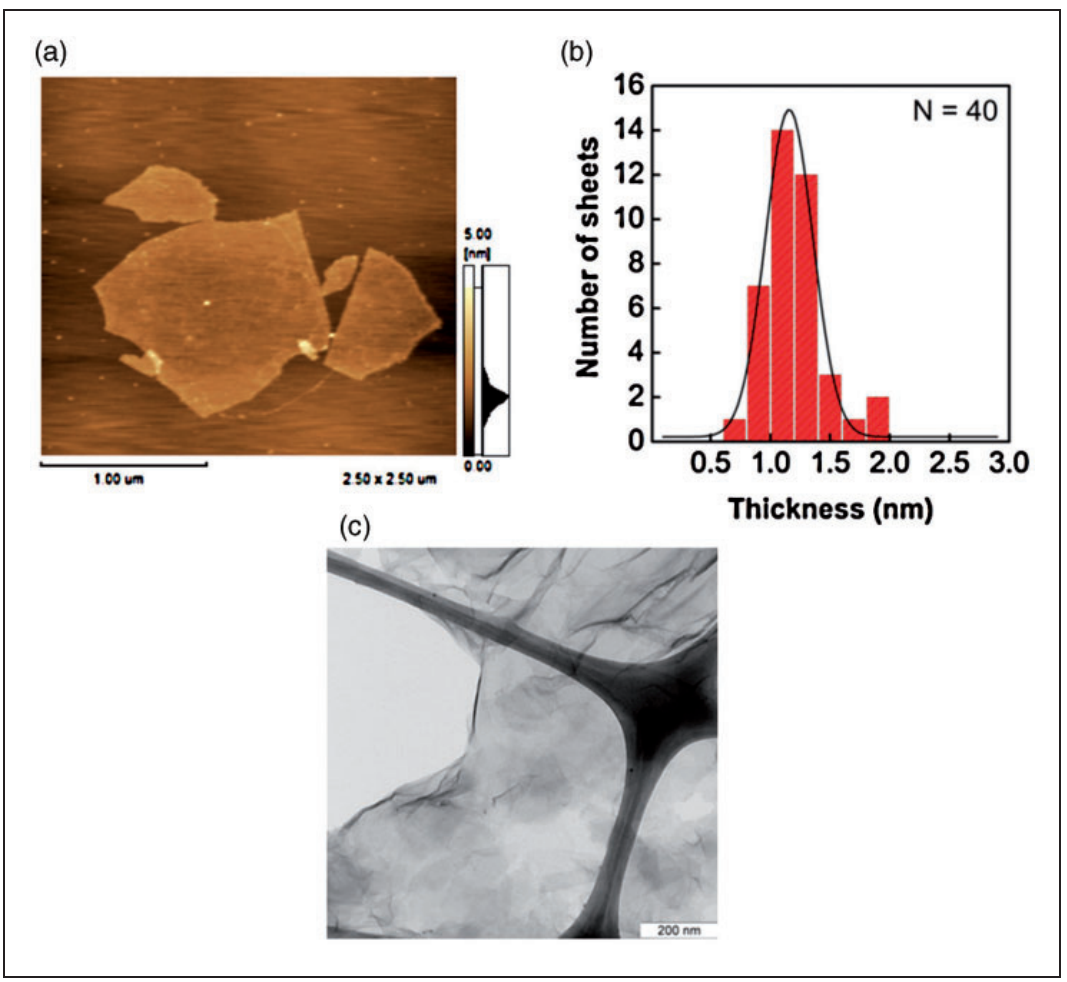

Figure 7. (a) Tapping mode AFM image of GO. (b) GO Sheet Height Histogram $(\mathrm{N}=40)$. (c) GO sheet TEM image. ${ }^{33}$

\section{Synthesis strategies}

The history of GO goes back many years to the earliest studies about graphite chemistry. Graphene oxide was first reported by Schafhaeutl in $1840^{36}$ and by Brodie in 1859. ${ }^{37}$ The British chemists were exploring the graphite structure by examining the reactivity of flake graphite. One of the reactions which he performed to synthesize graphene oxide, involved the adding potassium chlorate $\left(\mathrm{KClO}_{3}\right)$ to a graphite suspension in fuming nitric acid. He resolved that the resulting material contained hydrogen, carbon, and oxygen to enhance the total mass of graphite. The resulting crystalline materials have distorted structure, small size, and limited thickness. Therefore, the interfacial angle of the resulting crystal lattice could not be unable to calculated by reflective goniometry. Further the oxidative treatment increased the oxygen content reaching a limit after four successive reactions. The $\mathrm{C}: \mathrm{H}: \mathrm{O}$ composition was calculated to be 61.04:1.85: 37.11; having a net chemical formula 
$\mathrm{C}_{2.19} \mathrm{H}_{0.80} \mathrm{O}_{1.00}$. Brodie also noted that the material was easily dispersed in pure water but not in acidic medium. The material composition changed when heated to $220^{\circ} \mathrm{C}$ causing carbonic acid and carbonic oxide loss. The composition of this material changed to 80.13:0.58:19.29. He was interested in determining the discrete molecular weight of graphite. Finally, he determined the molecular weight of graphite to be $33 .^{37}$

Later in 1898, Staudenmaier improved the Brodie's synthesis method for graphene oxide by adding the chlorate manifold aliquots over the course of the reaction rather than in a single addition. ${ }^{38}$ This process is slightly changed from the Brodie's method and performed more practically in a single vessel. However, the overall oxidation level was similar Brodie's multiple oxidations approach. This method was the slowest and gave a lighter colored graphite oxide. However, it was both hazardous and time consuming because the $\mathrm{KClO}_{3}$ addition continued over a week. It was necessary to remove the chlorine dioxide release by using an inert gas, while explosion was a continuous hazard. Consequently, more modifications have been under investigation in this oxidation process. After Staudenmaier's approach, two chemists from Mellon institution of Industrial Research, Hummers and Offeman developed an alternate recipe for synthesizing GO. ${ }^{39}$ They prepared GO by oxidizing graphite with a concentrated water free sulfuric acid, potassium permanganate and sodium nitrate mixture. The graphite oxidation temperature was maintained below than $45^{\circ} \mathrm{C}$. According to their report, the whole oxidation process completed within $2 \mathrm{~h}$ and the final product has a higher oxidation level than Staudenmaier's product. The above mentioned methods differed little from one another but encompass the primary routes for making GO. Essentially, it has since been confirmed that the products of these three reactions showed strong discrepancy depending on the specific oxidizing agent used, the graphite source, and reaction conditions. Nitric acid is strong oxidant which is known to react with aromatic carbon surfaces of GO and carbon nanotube, resulting in forming different species containing oxide such as; carboxyl, ketone and lactone. $\mathrm{GO}$ oxidation by $\mathrm{HNO}_{3}$ may release the gaseous products $\mathrm{NO}_{2}$ or $\mathrm{N}_{2} \mathrm{O}_{4}$ in the form of yellow vapor as verified in Brodie's method. ${ }^{40}$ Similarly, potassium chlorate is also a common oxidizing agent and used in explosive materials such as blasting caps. Usually, $\mathrm{KClO}_{3}$ is an in-situ source to produce dioxygen, which acts as the reactive species. At that time, these were the strongest oxidation conditions and were used mostly on a laboratory scale. ${ }^{41}$ Flake graphite is a naturally occurring mineral and common raw material used to prepare GO including its oxidation. The flake graphite is purified to remove heteroatomic impurities such as iron and sulfur. ${ }^{42}$ It also consists of many localized defects in its $\pi$-structure that may act as best starting points for oxidation. However, the complicated 
graphite structure and inherent defects have rendered the elucidation of accurate oxidation mechanisms very challenging. Some other oxidizing agents for preparing $\mathrm{GO}$ have also been used. A mixture of $\mathrm{H}_{2} \mathrm{SO}_{4} / \mathrm{H}_{3} \mathrm{PO}_{4}$ is generally used for making expanded graphite, whose intercalated and partially oxidized structure is somewhere between true graphite and graphite oxide. ${ }^{43}$ Wissler et al. ${ }^{42}$ reviewed commonly used carbons and graphite fillers. In addition to these, they also described the terminology used to define these materials. $^{42}$

In 2010, Marcano et al. ${ }^{44}$ introduced a new a recipe for preparing GO. He used phosphoric acid for the reaction and also increased the $\mathrm{KMnO}_{4}$ in the absence $\mathrm{NaNO}_{3}$. The absence of $\mathrm{NaNO}_{3}$ was a big advantage for this procedure, as poisonous gases such as $\mathrm{NO}_{2}, \mathrm{ClO}_{2}$ or $\mathrm{N}_{2} \mathrm{O}_{4}$ were avoided. The reaction of graphite with $\mathrm{KMnO}_{4}$ in a mixture of $\mathrm{H}_{2} \mathrm{SO}_{4} / \mathrm{H}_{3} \mathrm{PO}_{4}$ (9:1) produced GO with higher extent of oxidation. Moreover, phosphoric acid was assumed to provide new intact graphitic basal planes and the final product was better much greater than Hummer's process.

In summary, these four different methods have been used to demonstrate:

- GO synthesis

- oxidation enhancement

- yield

- simplicity

- product quality.

Currently, producing GO for laboratory study is not a big problem, which thus has enabled GO research. However, the detailed understanding of the oxidation processes and their mechanisms are still challenging. Theses limitations may inhibit the reaction manipulation and chemical engineering to tackle some technology matters such as; size distribution control, band gap tuning, and edge structure selectivity.

\section{GO structure}

Apart from the effective oxidative mechanisms, the exact GO chemical structure has been debated over the years. Even today there is no definite GO structure model. The GO structure complexity may be due to different reasons. The reasons considered are:

- lack of precise analytical techniques for characterizing such materials

- complexity of the material (including sample-to-sample variability)

- berthollide character (i.e. nonstoichiometric atomic composition).

Although these GO properties make structure difficult to understand, reasonable efforts have been made to understand the structure with great success. According to the earliest structural models, $\mathrm{GO}$ is composed of regular 
lattices having repeated distinct units. Hofmann and Holst's structure proposed that across the basal planes of graphite consisted of spread epoxy groups (Figure 8). They think that $\mathrm{CO}_{2}$ is the net molecular formula. ${ }^{45}$ In 1946, Ruess ${ }^{46}$ suggested that along with epoxy groups, hydroxyl groups are also present in the GO basal plane. These groups are responsible for the hydrogen in GO. This Ruess model shows $\mathrm{sp}^{3}$ hybridization in GO basal plane structure, instead of $\mathrm{sp}^{2}$ hybridization proposed by Hofmann and Holst. The Ruess model still expects a repeat unit, where $1 / 4$ th of the cyclohexanes was composed of epoxides in the 1,3 positions and were hydroxylated in the 4 position making a regular lattice structure. Mermoux et al. ${ }^{47}$ postulated that the GO structure was similar to poly(carbon monofluoride), $(\mathrm{CF})_{\mathrm{n}}$, in which the complete rehybridization of the $\mathrm{sp}^{2}$ planes in graphite to $\mathrm{sp}^{3}$ cyclohexyl leads to the formation of $\mathrm{C}-\mathrm{F}$ bonds. ${ }^{48}$ Ten years later Scholz and Boehm ${ }^{49}$ reviewed the stereochemistry of this model and altered it to ridged carbon layers containing interchangeably linked ribbons of quinoidal structure and opened cyclohexane rings in chair conformation. They considered that there was no evidence for the presence of neither epoxy nor ether in the GO structure. Instead hydroxyl group was present on the 4 position of 1.2 oxidized cyclohexane rings. One useful model was proposed by Nakajima et al. ${ }^{50}$ In their model, they favored the lattice framework of GO similar to poly(dicarbon monofluoride), $\left(\mathrm{C}_{2} \mathrm{~F}\right)_{\mathrm{n}}$, leading to the formation of GIC.

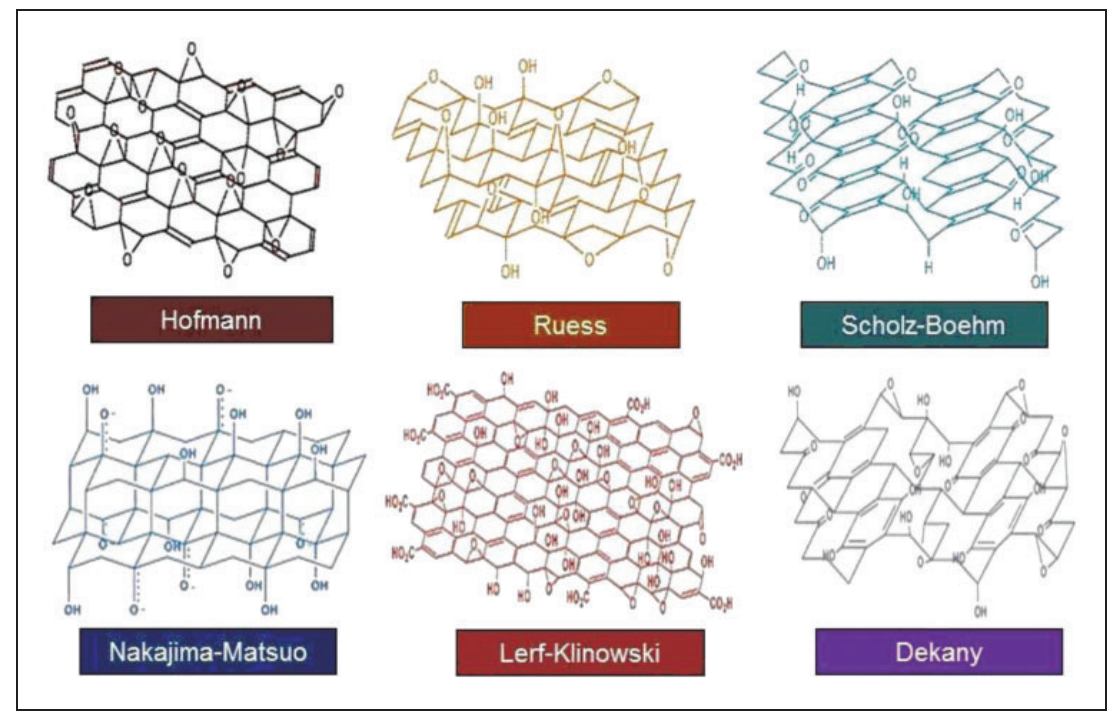

Figure 8. GO Structure. 
The above models were no longer important after the two recent models of Lerf-Klinowski ${ }^{51}$ and Dékány. ${ }^{52}$ According to Lerf-Klinowski et al., the GO structure was repeated non-stoichiometric amorphous and thus rejected the periodicity in structure. Firstly, they failed to prove Diels-Alder type cycloaddition reactions $(4+2)$ (conjugated double bonds should react) with maleic anhydride on GO. The steric effect of epoxy and hydroxyl groups present on GO surface may hinder its reactivity with dienophile. They thought that there might be alkene distributed on the GO basal plane. Later they recommended that alkene in GO may be aromatic or conjugated and that is why isolated double bonds should break during the severe oxidation conditions required for GO formation. ${ }^{51}$ They also suggested that keto groups were more favored at the edge of GO than carboxylic acids (FTIR). They explained that the GO acidity was due to tautomerization which is a reaction where there is a proton exchange converting the enol site to a keto site. Another well-recognized GO structure is the Dekany model, which accepted the logic in the Ruess and Scholz-Boehm models and favored the rigid nature of the carbon network. This model also followed the Scholz-Boehm model by adding 1,3-ethers in the GO structure which extends the trans-linked cyclohexyl networks. ${ }^{53}$ Cai et al. ${ }^{54}$ prepared a ${ }^{13} \mathrm{C}$-labeled GO sample in 2008 and analyzed it by ${ }^{1} \mathrm{D}$ and ${ }^{2} \mathrm{D}$ Solid-state nuclear magnetic resonance (SSNMR). These techniques concluded that only possible models were Lerf-Klinowski model and Dékány model. Concurrently ab initio chemical shift calculations were used to simulate the SSNMR signals in GO. Ab initio ${ }^{13} \mathrm{C}$-labeled GO geometry optimization and chemical shift calculations showed ${ }^{2} \mathrm{D}{ }^{13} \mathrm{C}$ double-quantum or single-quantum correlation. SSNMR spectrum was correlated with the spectra simulated for different structural models. These assumptions showed that only Lerf-Klinowski model was the best model with the experimental data, thus, all the earlier proposed models were omitted. ${ }^{55}$

\section{GO Physical properties and reactivity}

Dispersibility. Graphite oxide is a highly hydrophilic, strongly oxygenated layered material and easily exfoliated in water to give a stable dispersion. The dispersion contains single layer sheets which are also termed as graphene oxide sheets. ${ }^{56}$ Water is the most common dispersion media for GO, and there are two different ways to disperse GO in water including mechanical stirring and sonication. But sonication, in many applications, has been suggested to be less favorable than mechanical stirring because sonication generate defects and reduced the GO sheet size (from microns to a few hundred nanometers). ${ }^{57}$ Typically, the GO concentration in water is about $1-4 \mathrm{mg} / \mathrm{ml}$. The GO dispersion can also take place in many solvents such as ethylene glycol (EG), N-methylpyrolidine (NMP), N,N-dimethylformamide (DMF) and 
Tetrahydrofuran (THF). Thus, GO is believed to be amphiphilic in nature with the edges more hydrophilic and core more hydrophobic and may act like a surfactant. ${ }^{58}$ Paredes et al. ${ }^{34}$ noticed that GO could be dispersed nearly in all solvents excluding $n$-hexane, dichloromethane and to a smaller extent in $o$-xylene and methanol. Solvents such as ethanol, acetone, 1-propanol, pyridine, and DMSO showed limited dispersibility and all the GO particles settled in few hours. In contrast, GO dispersion in organic solvents has displayed long-term stability and very little precipitate was seen within a few days after sonication. UV-visible spectroscopy revealed that among the successful solvents, water provides highest absorption intensity and hence exhibits the best dispersing ability, and followed closely by DMF and NMP. ${ }^{59}$ It was observed that the above mentioned solvents show significant electrical dipole moment: water (1.82 D), DMF (3.24 D), NMP (4.09 D), THF (1.75 D) and EG $(2.31 \mathrm{D})$. On the other hand, solvents with small dipole moment (o-xylene, $0.45 \mathrm{D}$ and $n$-hexane, $0.085 \mathrm{D})$ completely failed to disperse GO. Though, there are some solvents with high electrical dipole moment (DMSO, 4.09 D) fail to provide long term dispersion stability. Therefore, it is suggested that beside solvent polarity other factors are important which determine the good dispersibility. ${ }^{60}$

Toxicity. Akhavan and Ghaderi ${ }^{61}$ reported that GO and reduced GO (RGO) are more toxic to bacteria (Staphylococcus and E.coli) due to bacteria cell membrane damage when in contact with the edges of GO and RGO. He also studied that the GO reduced by hydrazine was more toxic than pristine GO. $\mathrm{Hu}$ et al. ${ }^{62}$ also showed that GO is slightly toxic towards human alveolar epithelial cells and may inhibit the bacterial growth. Wang et al.$^{63}$ revealed that the GO toxicity was dose-dependent and when the dose was higher than $50 \mu \mathrm{g} / \mathrm{ml}$, damage was observed towards human fibroblast cells. Blood compatibility and GO toxicity were described to be dependent upon exfoliation level, dose and GO sheet size. Zhang et al.$^{64}$ demonstrated that RGO was less toxic than carbon nanotube toward $\mathrm{PCl}_{2}$ cells at high concentration. Ruiz et al. ${ }^{65}$ reported these discrepancies and confirmed that GO is non-toxic to both mammalian and bacterial cells. The study ascribed that the above reported toxicities were due to impurities in GO. However, the incorporation of Ag nanoparticles into GO matrix considerably increased its antibacterial activity.

Intercalation with water (hygroscopicity). Graphene oxide is very hydrophilic due to oxygen containing functionalities such as; hydroxyl, carboxyl and epoxy and absorbed water molecules in the interlayer sheet spaces, even after long drying. ${ }^{66}$ Therefore, GO is quite hygroscopic in nature which strongly depends upon the humidity. When water molecules are present in large 
extent in the interlamellar spaces in a stacked GO films, a strong hydrogen bonding network is formed between oxygenated functionalities on GO and water molecules. So mechanical, structural and electronic properties were significantly influenced. ${ }^{67}$ For instance, when the humidity level increases, the GO film swells and tensile modulus decreases. Few theoretical simulations predicted that when the water content increases from zero to $26 \%$, the distance between the interlayer sheets of GO flakes increases from $5.1 \AA$ to $9.0 \AA$. Moreover, when the water content surpasses $15 \%$, the H-bonding network is dominated by water-water H-bonds, whereas the functional groups are connected indirectly through a chain of water molecules. ${ }^{68}$ Fascinatingly, a lower interlayer distance between GO sheets is observed, when water content is replaced by $\mathrm{D}_{2} \mathrm{O}$. This was probably due to lower reactivity, stronger bonding, and lower $\mathrm{D}_{2} \mathrm{O}$ solubility compared with water. During the reduction process of GO, the hole and carbonyl formation have also been accounted due to the presence of water. ${ }^{69}$

Graphene oxide reactivity. GO reduction is mainly considered in terms of its chemical reactivity, so reducing agents were used for this purpose. Hofmann in 1934 used $\mathrm{H}_{2} \mathrm{~S}$ as a reducing agent. ${ }^{70}$ Although lithium aluminum hydride is strong reducing agent, it is not used to reduce GO due to strong reactivity with $\mathrm{GO}$ dispersion medium. While $\mathrm{NaBH}_{4}$ reacts slowly with water its reaction rate is rapid enough for reduction. Beside all reducing agents, hydrazine derivative has been the most useful for GO reduction. ${ }^{71}$ Strongly alkaline and supercritical water environment can also be used for GO reduction. ${ }^{72}$ Chemical methods have some disadvantages in introducing impurities. For example, some hydrazine derivatives tend to covalently attach to nitrogen, along with removing oxygen from GO. ${ }^{73}$ Heating GO above $100^{\circ} \mathrm{C}$ directly in furnace can also reduce it. This high temperature reduction method is known as thermal method, where 30\% GO loss was observed. Main defect in thermal reduction method for GO was some structural distortion. The structural distortion might affect GO physical properties. ${ }^{74} \mathrm{~A}$ third method to remove oxygen from GO is an electrochemical method which leads to changing the GO structure. Basic approach in this method was GO thin film deposition on different substrates. The electrode was placed opposite to the thin film and potential was applied to the buffer solution. ${ }^{75} \mathrm{GO}$ chemical fictionalization can also be done by adding both covalent and noncovalent entities. Amines, esters, thionyl chloride, poly(vinylalcohol) and octadecylamine have been mostly used for covalent functionalization of GO. This functionalization was usually seen at carboxylic acid groups ${ }^{76}$ and also at epoxy group especially in case of ionic liquid. As van der Waals forces along with $\pi-\pi$ interaction are present in GO, so there was strong 
evidence that non-covalent functionalization is present. Yang et al. ${ }^{77}$ were the first to prove this. They prepared graphene oxide/doxorubicin hydrochloride (DXR) hybrid via non-covalent interaction. They also proposed that strong hydrogen bonding existed between the $-\mathrm{NH}_{2}$ and $-\mathrm{OH}$ in $\mathrm{DXR}$, and $-\mathrm{CO}_{2} \mathrm{H}$ and $-\mathrm{OH}$ groups of $\mathrm{GO}$.

\section{GO paper}

Carbon nanofillers are used in many applications due to their remarkable electrical, thermal and mechanical properties which have been determined both theoretically and experimentally. The history of bucky paper starts in 1985, when Harry Kroto, a British scientist, found elemental carbon a key building block of life. They discovered the buckyball composed of 60 carbon atoms also named buckminsterfullerene. ${ }^{78}$ In 1996, Harlod Kroto and Robert Curl were awarded a Nobel Prize for chemistry. Later on, at Smalley's laboratory researchers found that tubes in a liquid suspension stick together upon dispersion. They filtered the suspension via fine filter support producing a thin film known as buckypaper. The buckypaper was made from randomly entangled CNT sheet through a vacuum filtration method or other papermaking techniques. The SEM images show that the buckypaper has a porous structure and consisted of randomly interconnected CNT (Figure 9). ${ }^{79}$ The buckypaper (BP) membrane thickness was $10-15 \mathrm{~mm}$ had low density and was mechanically strong due to high surface area. Electrical and mechanical properties of buckypaper render them a favorable material for producing novel composites. Similar to CNT buckypaper, graphene oxide can also be

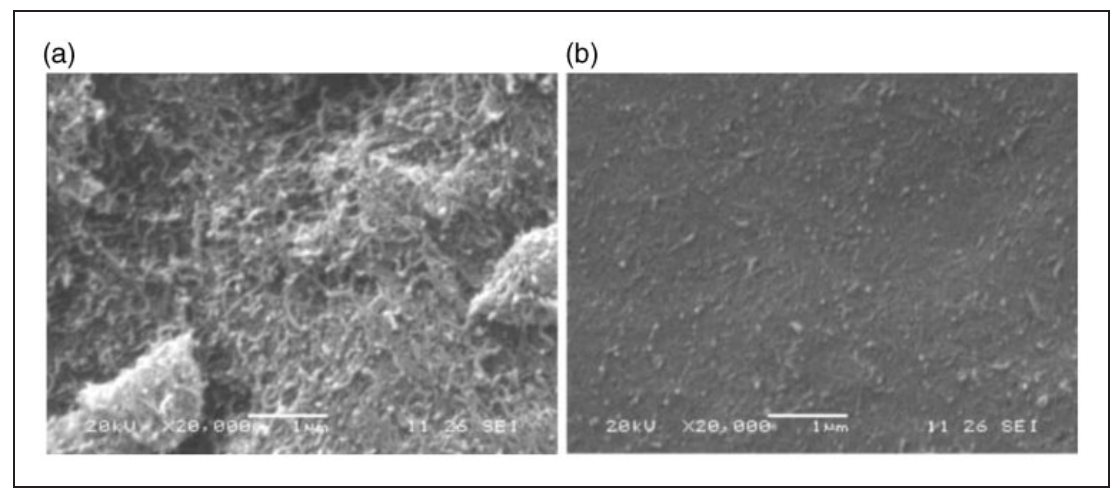

Figure 9. Neat buckypaper SEM photographs (a) the matt surface; (b) the opposite surface. $^{79}$ 
assembled into paper-like materials under direction flow via a simple vacuum filtration method or evaporation induced self-assembly. The GO paper is a self-supporting thin film that consists of aligned GO nanosheets. The wellordered GO paper exhibits outstanding mechanical properties such as up to $40 \mathrm{GPa}$ young modulus and up to $125 \mathrm{MPa}$ tensile strength which surpass many other paper-like materials such as graphite foil. ${ }^{80}$ The GO paper has a layered structure and is composed of individual, well-organized 100-200 nm thick sheets (Figure 10).

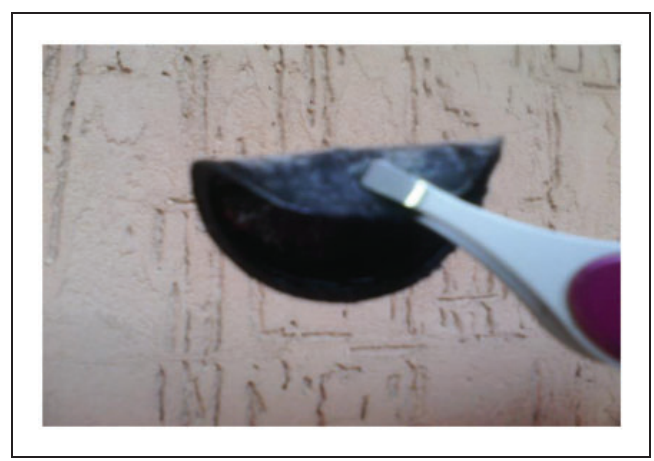

Figure 10. Graphene oxide paper.

The plane and edges of graphene oxide have oxygenated functional group and produce $\mathrm{sp}^{2}$ and $\mathrm{sp}^{3}$ hybridized carbon atoms. It results in better

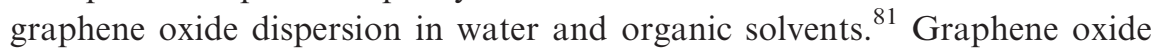
applications are mainly in energy related materials, building block in composites, nano-robots, mechanical actuators, medical, and biological applications. ${ }^{82,83}$ Recently, due to outstanding properties like its high flexibility, modulus and strength, GO-based paper materials have attracted much attention. ${ }^{84}$ The GO-based paper-like materials may be used as supercapacitors, sealants, bio-compatible substrates, actuators, and flexible substrates with high thermal and chemical stability. ${ }^{85}$ Free standing layer-by-layer hierarchical structure of graphene oxide paper can easily be prepared from an aqueous graphene oxide dispersion using a vacuum-assisted self-assembly process. Figure 11 shows the morphology and TEM images of GO papers. The graphene oxide paper mechanical properties have been usually studied in bending modes and unidirectional tensile. ${ }^{66}$ The individual graphene oxide nanosheets with their interlocking-tile microstructure is the reason graphene oxide paper has high strength and modulus. The SEM image in Figure 12 shows the layer structure of GO paper. ${ }^{86}$ 


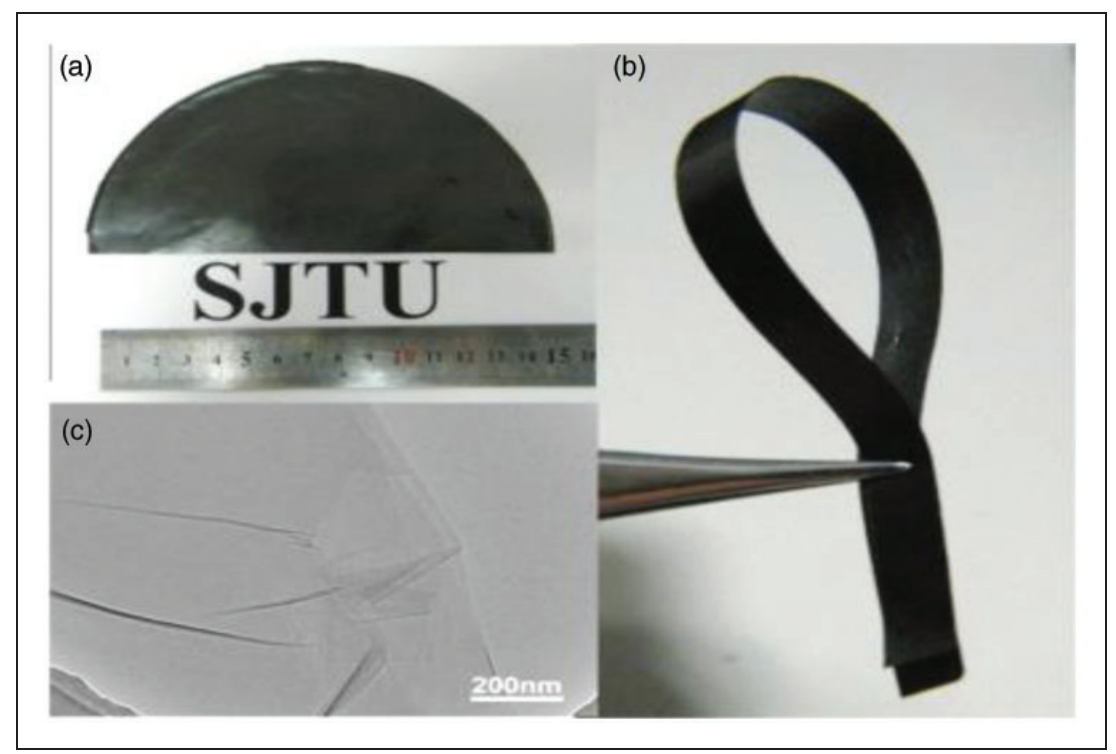

Figure II. GO paper morphology (a) and (b); (c) TEM image. ${ }^{86}$

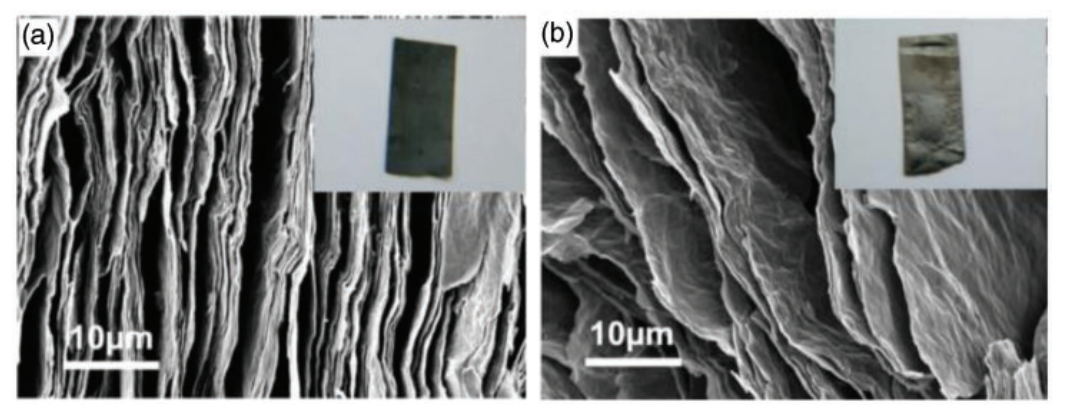

Figure 12. GO paper SEM images and Digital photo (Insets). (a) Pristine sample; (b) after DMA measurement at $150^{\circ} \mathrm{C}^{86}$

While the graphene oxide paper properties are different from other paperlike materials, ${ }^{87}$ they are similar to carbon nanotube and flexible graphite foils. ${ }^{66}$ The difference in graphene oxide paper properties is dependent on many factors such as precursor materials and how they are produced. 
For example, the modulus is between 6 and $42 \mathrm{GPa}$ and tensile strength varies between 76 and $293 \mathrm{MPa}^{88}$ Intercalation of polymer layers and chemical cross-linking between the graphene oxide sheets can be used for improving the mechanical properties of GO papers. This technique may be used for the intercalation of divalent ions, such as $\mathrm{Ca}^{+2}$ and $\mathrm{Mg}^{+2},{ }^{66}$ glutaraldehyde (GA) and water molecules, ${ }^{89}$ polyvinyl alcohol films, ${ }^{90,91}$ polydopamine (pDOP), ${ }^{92}$ octadecyclamine (ODA), ${ }^{86}$ and the combination of pDOP and polyetherimide (PEI) ${ }^{88}$ Stiffness (10-200\%) and strength (50\%) of GO papers were enhanced by modifying with $\mathrm{Ca}^{+2}$ or $\mathrm{Mg}^{+2}$ (less than $1 \mathrm{wt} . \%$ ). ${ }^{87}$ pDOP was poured into the suspension during filtration, ${ }^{92}$ while graphene oxide suspension was used in a buffered aqueous solution at basic condition. The significant alignment with a planar structure of GO sheets and pDOP was allowed by this process. Due to the in-situ reduction of graphene oxide by pDOP, the composite paper showed elongation and increased tensile strength including recovered electrical conductivity. Recently, graphene oxide sheets have been cross-linked by a pDOP and PEI mixture. The fracture strength of the covalently cross-linked papers was double the original GO papers. Their Young's modulus improved by $5 \%$, which was the highest reported in the literature. ${ }^{88}$ Polymer cross-linking efficiency has been influenced by PEI and the external stress applied in the crosslinking process. A novel thought based on electron irradiation was also effectively used to cross-link the GO layers. ${ }^{93}$ The remaining oxygen containing functional groups were dislodged during irradiation with associated reduction in interlayer spacing. Improvement in strength and tensile modulus was observed up to $48 \%$ and $73 \%$ at an optimum irradiation dose. The above studies indicated that the fracture properties of GO papers in other modes of deformation, such as in shear or tearing were very low. Water strongly affected the strength and modulus of GO papers. ${ }^{94}$ When the external force was applied, interlamellar water molecules reorient causing lower modulus of GO paper. ${ }^{68}$ Water content had a softening property on tensile strength of graphene papers, so limiting their modulus and strength while increasing the low strain. ${ }^{91}$ The SEM analysis of the fracture surface of vacuum filtrated GO and GO/PVA papers (Figure 13) showed that it contain well-aligned GO flakes. Theoretically water may slightly raise the modulus and strength via improved interlayer bonds. ${ }^{95}$ Small and large GO sheets are perfectly suitable for many applications. For instance, large GO sheets with controlled size were preferably suited for optoelectronics devices; while polymer-based nanocomposites with small GO sheets were preferable for drug delivery and bio-sensing. However, to control the GO size or to obtain GO with large size, various techniques have been suggested including sonication and less oxidation. 


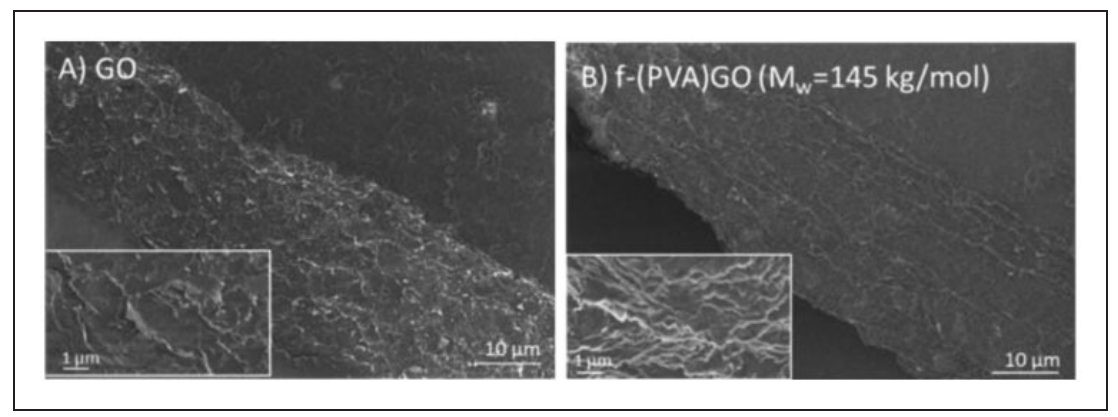

Figure 13. SEM fracture surfaces of vacuum filtered paper-like materials: (a) GO (b) f-(PVA)GO. ${ }^{91}$

\section{GO paper fabrication techniques}

To produce graphene and graphene oxide papers, different methods have been used such as; vacuum filtration assembly, ${ }^{96}$ evaporation induced self-assembly, ${ }^{97}$ spin coating, ${ }^{98}$ drop-casting, ${ }^{99}$ Langmuir-Blodgett (L-B), ${ }^{100}$ spraying, ${ }^{101}$ dip coating ${ }^{102}$ and Langmuir-Schaefer. ${ }^{103}$ Controlling the surface morphology, film uniformity, surface coverage and thickness of the paper depends upon the deposition methods and other parameters used. Spraying, dip coating, and drop casting, due to GO aggregation on substrate surface, frequently results non-uniform deposition and allows poor film thickness control. Once the GO or RGO are deposited, the GO sheets are adhered strongly to the substrate via van der Waals forces. ${ }^{104}$ The individual GO sheets are also held together through strong hydrogen bonding which helps the films or papers to adhere with hydrophilic surfaces. To encourage the thin film deposition on glass substrate, it is necessary to treat their surface with acid or (aminopropyl) triethoxysilane before deposition. ${ }^{105}$

\section{Vacuum filtration method}

The most frequently used technique to fabricate $\mathrm{GO}$ free standing papers and GO films is vacuum filtration. This method has also been utilized to produce highly uniform thin carbon nanotube buckypaper for flexible and transparent electronic devices. ${ }^{106}$ To achieve thin GO paper, the GO powder is first dispersed in water or any other organic solvent either by sonication or by mechanical stirring to get a GO suspension. The suspension with comparatively low GO content $(0.5 \mathrm{mg} / \mathrm{l})$ is then filtered through a porous support either mixed cellulose ester membrane or polycarbonate membrane (Figure 14). While filtering the suspension through the porous membrane, the solvent flow is enhanced with the help of vacuum pump. As a result, the GO sheets 


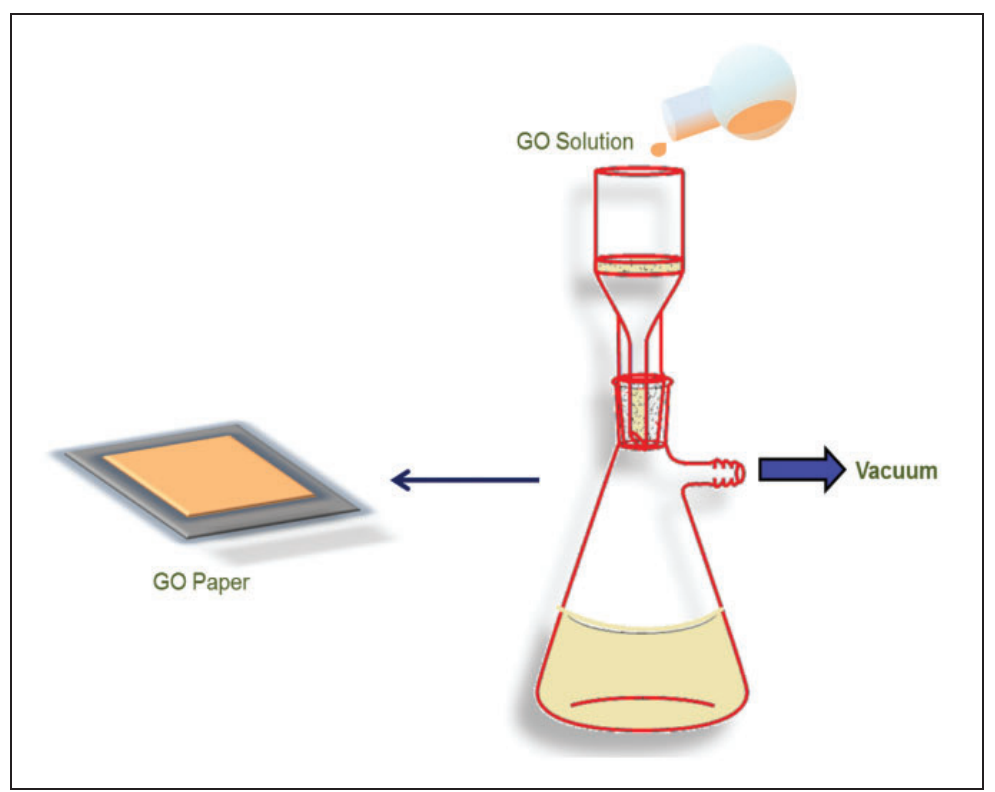

Figure 14. GO paper prepared via vacuum filtration.

stay on the membrane and form paper like materials. The GO paper is then peeled off from the porous membrane and dried. The paper or films thickness is controlled readily with nanoscale precision by volume filtered and the graphene oxide concentration. This process also allows good percolation of GO sheet in sub-monolayers and controls the film thickness at nanometerscale range. ${ }^{107}$ Multiple techniques have been used to transfer the GO films on to many substrates by pressing gently against the substrate surface. The GO films can be made free-standing over the considerable openings $\left(\sim 1 \mathrm{~cm}^{2}\right)$ by transferring the GO film to the substrate with an aperture. The film is placed over the substrate before dissolving the membrane. ${ }^{108}$

\section{Spin coating method for GO paper or film}

Spin coating is used to deposit GO suspension on the flat surface. For this purpose, relatively high GO concentration is required to make thin uniform continuous film. The apparatus used is a spin coater, where the substrate is rotated at relatively high speed and the materials spread on the surface via centrifugal force. ${ }^{109}$ The solvent used is volatile and evaporates simultaneously. During spinning, rapid solvent evaporation results in greater interaction between the substrate surface and GO sheets, thus increasing adhesion. Figure 15 shows the steps. The film thickness can be controlled by RPM, 
GO concentration and solvent viscosity. Thin uniform GO film deposition has been demonstrated on $30 \mathrm{~cm}$ wafers through carefully controlling solvent evaporation rate. ${ }^{110}$

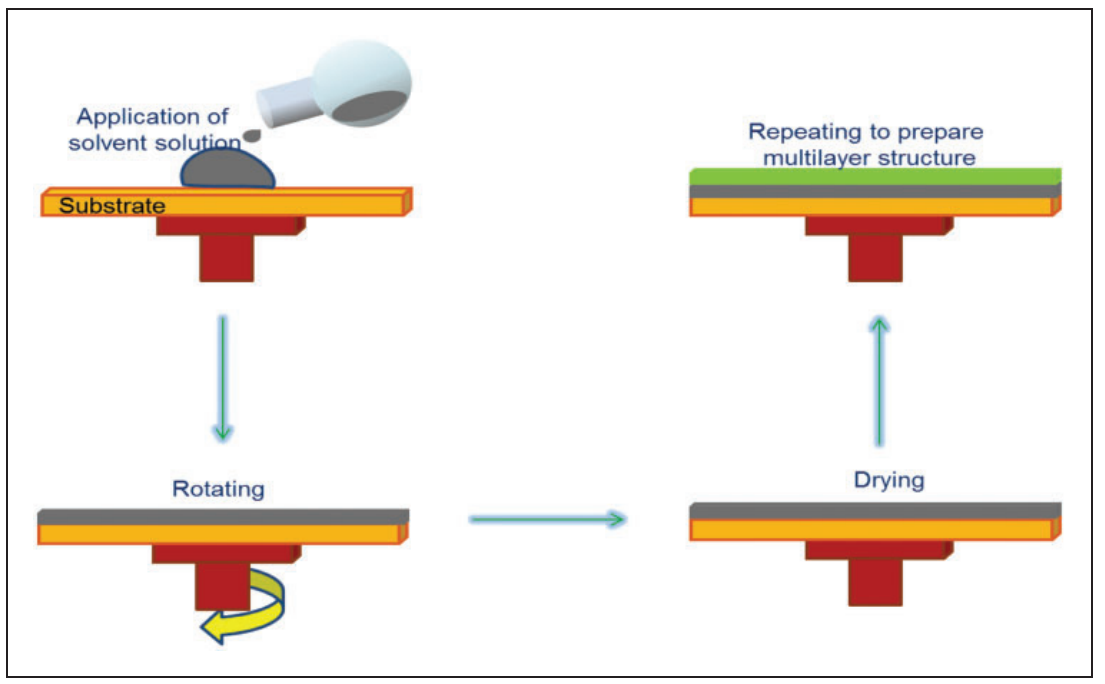

Figure I5. Spin coating technique for GO multilayer structure.

\section{Langmuir-Blodgett (L-B) assembly}

The technique was invented by Langmuir and Blodgett. ${ }^{111}$ It was used to deposit one or few monolayers of GO sheets on a solid substrate in order to fabricate highly uniform GO film. This L-B assembly exploits the electrostatic repulsion between the functional groups present particularly on the ionized edges of GO sheets. Briefly, the GO suspension in a methanol and water mixture was spread carefully over the liquid surface to obtain the floating sheets of GO trapped at the liquid/air interface. Then, the solid substrate was immersed into the liquid and from the liquid surface the floating GO sheets were deposited on the solid substrate (Figure 16). The density of floating sheets of GO can be controlled by changing the available area of liquid/air interface. Since the sheets were stabilized electrostatically, they continue to disperse as monolayers with reducing area until the attractive forces between sheet faces are overwhelmed by the repulsive forces. These repulsive forces between GO sheets may lead to layer stacking. ${ }^{100}$ Robinson et al. ${ }^{108}$ demonstrated the transfer of $\mathrm{GO}$ thin film from $\mathrm{SiO}_{2} / \mathrm{Si}$ substrates onto the other substrate through delamination in water. He treated the GO films with sodium hydroxide solution and dipping subsequently in water leading to 


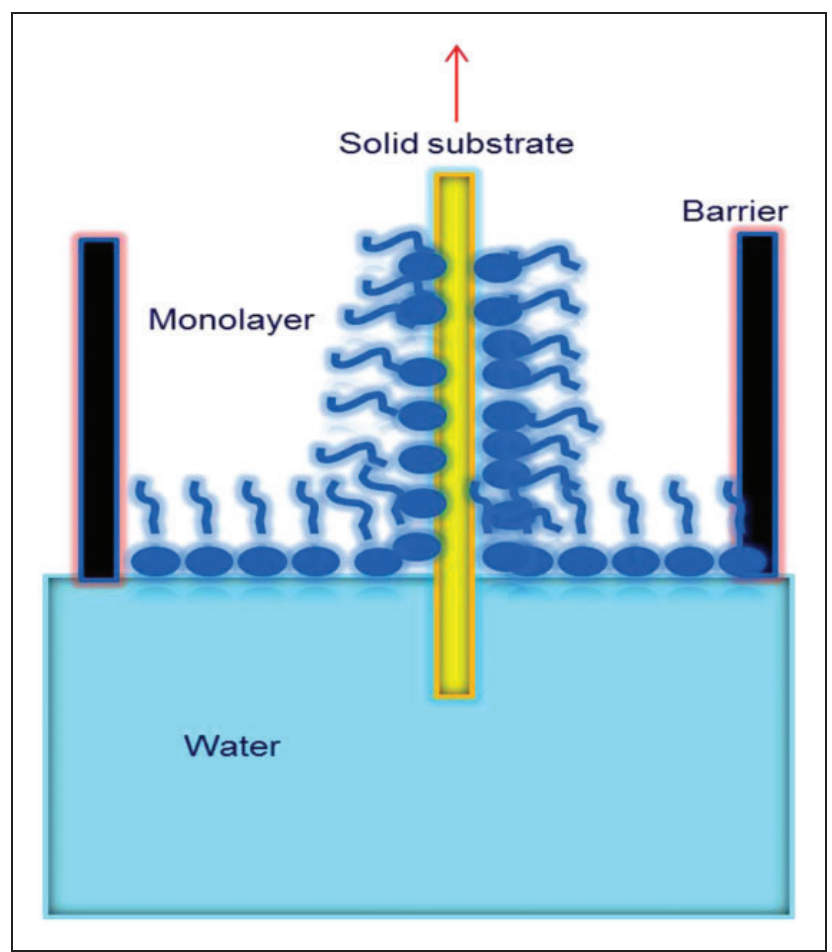

Figure 16. Langmuir-Blodgett assembly for GO monolayer structure.

uniform delamination of GO film floating on the water surface. Then the film can be transferred onto another desired substrate. During the transfer, an additional polymer layer can be deposited in order to control the GO film disintegration into individual sheets. L-B assembly is suitable for producing close-packed, highly uniform monolayered GO film in the subpercolation regime, while the films produced by spin coating are mostly highly continuous and cover the whole substrate. Vacuum filtration produced film or paper with variable thickness and frequently with obvious wrinkling, particularly for thick films. ${ }^{12,113}$

\section{Resin infiltration for polymer/GO composite paper}

There are two steps to prepare polymer/GO composite paper by vacuum resin infiltration. The first have GO paper prepared previously by vacuum filtration, while the second step is casting of polymer solution over the GO paper. The GO powder is dispersed in organic solvent either by stirring or by sonication and GO suspension is obtained. Then this suspension is filtered 
through a cellulose membrane with a pore size $0.45 \mu \mathrm{m}$ by vacuum filtration assembly. GO paper is obtained on the surface after passing the entire solvent through a porous membrane. Then, the polymer solution is infiltrated upon GO paper. After passing the solvent, the polymer/GO composite paper is obtained, dried and peeled off from the porous membrane. ${ }^{106}$ Figure 17 shows the preparation of polymer/RGO composite film by vacuum filtration.

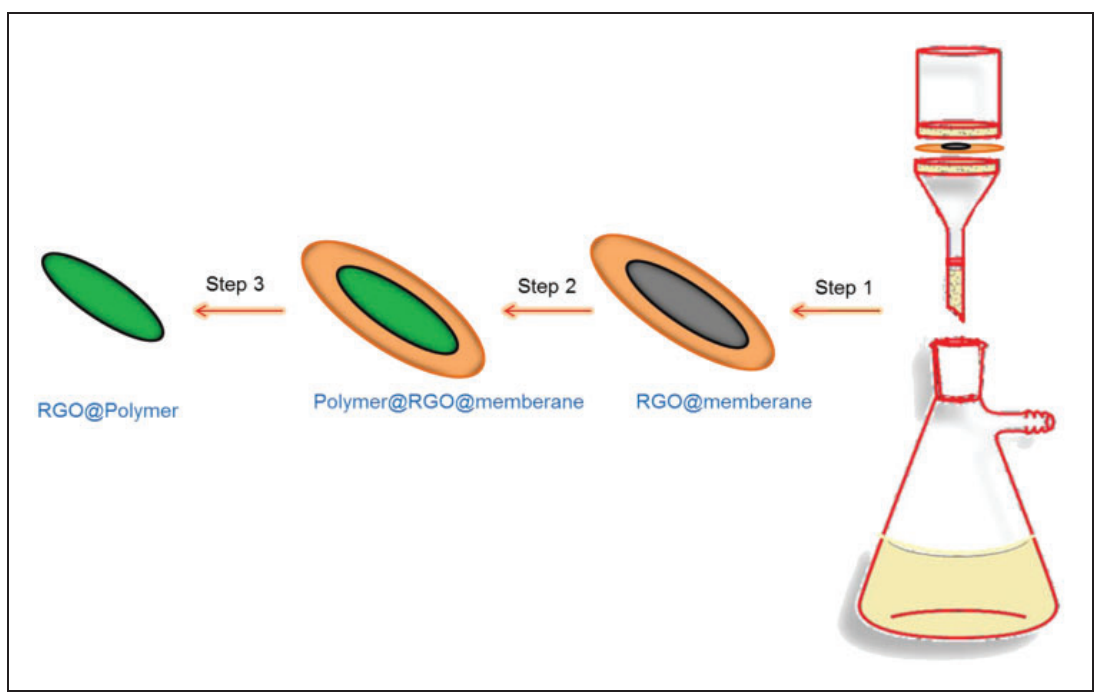

Figure 17. Polymer/RGO composite paper preparation via resin infiltration.

Firstly, RGO film was deposited on porous membrane by vacuum filtration and dried at $60^{\circ} \mathrm{C} .^{112}$ The film was then coated by polymer (PDMS or PVA) by cast coating or spin coating and polymer/RGO composite film were obtained. Then, the coated polymer film was dried at $80^{\circ} \mathrm{C}$. The resulting polymer/RGO film was removed from membrane in the process reported by Wu et al. ${ }^{13}$

\section{Evaporation-induced, self-assembly (EISA)}

Evaporation-induced, self-assembly is well-organized, unique and facile selfassembly method used to produce free-standing, macroscopic Ag-reduced GO (Ag-RGO) films. ${ }^{114}$ This method is a flexible and powerful process for preparing well-ordered thin GO Janus film. Moreover, the EISA based Janus films provided a new route for materials based on graphene. As a result of surface charge distribution or asymmetric shape, Janus films were expected to have exceptional properties. Although preparing Janus a film was an unexplored area and there were many reports on the preparation and uses of Janus 
particles, cylinders and vesicles. ${ }^{115}$ The synthetic route to fabricate Janus films based on EISA has the following steps:

- The aqueous precursor solutions were poured into a Teflon dish containing GO and Tollen's reagent

- Then these solutions underwent EISA process

- Following the water evaporation an ultra-thin monolayer film appeared on the mother liquor surface.

- The continuous water evaporation stimulated the GO and Tollen's reagent assembly into the preferred laminated geometry. Then, these species hardened into well-organized GO and Ag layers.

- Next, the films were treated with hydrazine to remove the oxygen containing functional groups, which also restored the $\mathrm{sp}^{2}$ network and reduced the silver species.

- Finally, the desired Janus film was obtained, which could be selfassembled further on a different substrate such as glass, silicon chip, quartz, and certain polymers. ${ }^{114}$

\section{Polymer/GO composite papers}

Free standing graphene oxide paper materials showed remarkable mechanical stiffness and excellent flexibility combined with outstanding electrical conductivity. Therefore, these properties render the GO paper potentially suitable for flexible electrochemical active materials such as; electrical batteries, fuel cells, biomedical devices, and supercapacitors. ${ }^{116}$ However, less-active unitary graphene surface and less electrical conductivity of GO inhibit them from undergoing better electrochemical activity. Hence, including an electrochemically active and an electrically conductive second phase into the GO and graphene paper materials is essential to improve their electrochemical properties. Adding graphene oxide filler materials into a polymer matrix can enhance the resultant nanocomposites properties, both mechanically and thermally, as compared to the pure polymer. Incorporating GO at low concentration into a polymer matrix can totally change the polymer chemistry producing new properties including fire-retardant characteristics and antimicrobial properties. ${ }^{117}$ Recently, a favored conductive polymer polyaniline (PANI) has been used. Due to their low cost, controllable conductivity, highly electrochemical activity, and good biocompatibility combined with the easy preparation, PANI composites materials have been extensively considered as electrochemical, electrical, and biomaterial. ${ }^{118}$ Recently, synthesis of graphene-PANI and GO-PANI composites papers have been explored which are likely to display many functional properties. Particularly, PANI has been studied to promote the electrochemical capacitance of graphene and GO paper-like materials. ${ }^{119}$ It is recognized that GO papers contain 
carboxylic and phenolic hydroxyl functionalities. When immersed in polymer aqueous phase, the aniline monomer gets adsorbed on the site with the abovementioned functional groups through van der Waals forces or hydrogen bonds. Therefore, these sites perform like seeding dots forming polyaniline nanoparticles. ${ }^{120}$ As compared to parent GO and graphene composites papers, the obtained hybrid GO-PANI and graphene-PANI papers exhibited outstanding biocompatibility and excellent electrochemical properties. These characteristics make them attractive for novel applications in biomedical sciences such as; biosensors, cell culture, drug delivery, and electroactive substrate for tissue engineering. ${ }^{121}$ Several techniques such as cyclic voltammetry (CV), galvanostatic charge/discharge, and electrochemical impedance spectroscopy (EIS) were used to analyze the electrochemical performance of the $\mathrm{GO}$ paper-like materials. From the $\mathrm{CV}$ curve, the capacitance was reported by integrating over full $\mathrm{CV}$ curve in order to determine the average value. ${ }^{122}$

The hydrogen bond network plays a key role in determining the whole morphology and mechanical strength of GO paper. Due to excellent mechanical properties, GO paper has attracted great interest in novel applications such as; transparent paper-like materials, composites, mechanical actuators, and nanoelectrochemical systems. When the water content in GO paper was negligible, the interlayer distance was $5.1 \AA$. However, the interlayer distance increased to $9.1 \AA$ when the water content increased to $26 \% .{ }^{123}$ It means that the difference in interlayer distance between GO layers did not depend on their chemical composition but only depend on the water molecule concentration present between the GO layers. Thus, depending on the relative humidity, the typical interlayer distance was reported to increase between 5.1 and $9.1 \AA^{124}$ In composite GO paper, three H-bonding states are present (Figure 18):

- intralayer H-boding: H-bonds between oxygen functional groups attached to the same graphene layers

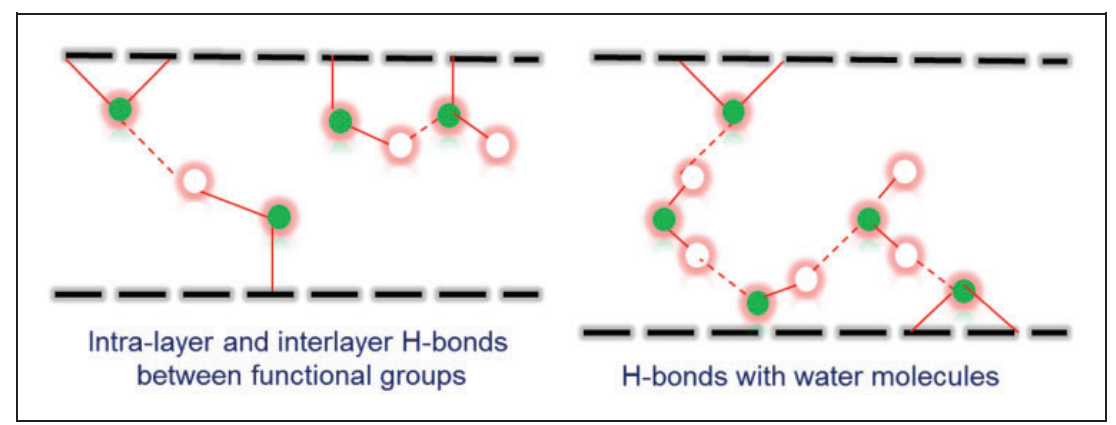

Figure I8. GO layer hydrogen bond network without and with water molecules present. 
- interlayer H-bonding: H-bond between oxygen functional groups attached to the adjacent graphene layers

- H-bonding between oxygen functional groups and water molecules.

When the hydration level is high, the H-bonds between water and functional groups dominate, therefore, the GO layers are well-separated. When the hydration level is high, the intra and interlayer $\mathrm{H}$-bonding between oxygen functionalities dominate. So, the distance between the interlayers is small (5-6 $\AA$ ) and the GO composite structure is very compact. Therefore, the GO papers with no water molecules are stiffer and more compact structurally than the GO papers containing water molecules. ${ }^{68}$

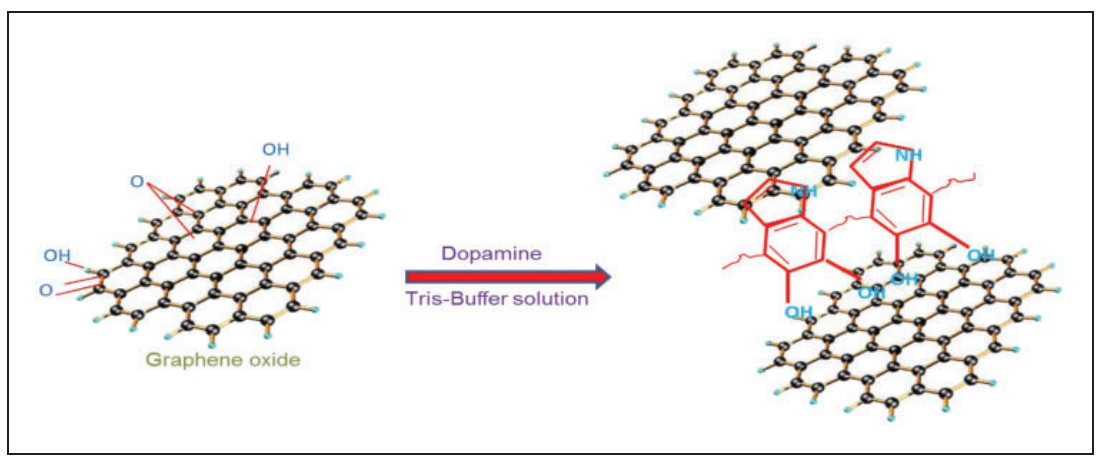

Figure 19. Graphene oxide coated while being reduced by dopamine.

Liang et al. ${ }^{125}$ reported synthesizing PVA/GO composite paper via a water solution processing method. They suggested that a strong H-bonding network between functional groups on GO layer and PVA OH groups determined the molecular level distribution between PVA and GO layers. Due to which the composite mechanical properties were improved considerably. The GO/PVA composite paper tensile strength and Young's modulus increased up to $76 \%$ and $62 \%$, respectively, by the adding $0.7 \mathrm{wt} . \%$ GO filler. Lee et al. ${ }^{126}$ used Dopamine in order to enhance the mechanical properties of GO paper. Dopamine is an excellent adhesive in polymeric forms and has very strong adhesion to all inorganic and organic surfaces similar to covalent bonding and considerably higher than H-bonding. Materials comprising dopamine have excellent adhesive ability and have been used as effective reinforcing agents. ${ }^{127}$ When dopamine was intercalated between the GO sheet layers during the filtration, dopamine self-polymerization occurred under the basic buffer condition. Figure 19 shows polydopamine (pDop) formed between the individual GO layers. Due to intercalation of pDop, a strong adhesion force was produced between the GO layers which replaced hydrogen bonding and 
weak van der Waals forces. Therefore, the pDop intercalation increased the graphene paper mechanical strength. In contrast with GO paper, both elongation and mechanical strength of pDop-reduced GO paper were enhanced by $70 \%$ and $35 \%$, respectively. This indicates that the adhesive ability of pDop in reduced GO paper was stronger than hydrogen bonding in GO paper. Hence, well-intercalation of pDop in GO layer effectively improved the mechanical, thermal, and electrical properties. ${ }^{92}$

\section{Polymer/graphene composite paper}

Graphene is an innovative material and due to its excellent physicochemical properties it has been widely used in various research areas. Graphene has infrequent electronic properties such as high carrier mobility at room temperature. The charge carrier concentration is comparatively high and an anomalous quantum Hall effect is due to its exceptional electronic structure. ${ }^{128}$ Graphene also displays enormous oxygen containing functional groups and active edges. It has excellent mechanical and electrochemical properties as compared to carbon nanotube. ${ }^{129}$ Flow-directed assembly has already been used to fabricate flexible papers containing GO sheets or graphene sheets as sole building units. Graphene papers exhibit room temperature electrical conductivity of $7200 \mathrm{Sm}^{-1}$ and remarkable tensile strength up to $35 \mathrm{GPa}$ (5 million psi). ${ }^{130}$ These fascinating properties enable graphene paper to be a freestanding electrode. In addition to these, several conducting polymers (because of low cost, easy production and high capacitance) have been studied extensively as electrode materials for supercapacitors. Though, weak flexibility and poor conductivity of conducting polymer limit their use in high performance supercapacitors. However, it has been predicted that inserting graphene into the polymer not only improves the mechanical strength but also particularly improves the electric conductivity of polymer composite papers. ${ }^{131}$

Recent research on graphene was originally focused on its infrequent electrical transport properties and potential future applications in nanoelectronics. Graphene sheets, due to their high electrical conductivity and remarkable mechanical and thermal properties, have also found great interest as nanoscale building blocks to produce exceptional macroscopic materials. ${ }^{132}$ In recent years, graphene sheets have been synthesized in large amounts via chemical conversion of graphite, which has enabled fabricating innovative graphenebased electronic devices. ${ }^{101}$ It was also possible to produce new bulk materials including graphene sheets for various technical applications. Recently, conducting polythiophene/graphene nanocomposites has been developed by Shah et al. ${ }^{133}$ The polythiophene was prepared using in-situ chemical oxidative polymerization. The simple sonication in a protic solvent was used to convert graphite to graphene. The polythiophene/graphene nanocomposites were 
electrically conductive $\left(1.8 \times 10^{-3}\right)$ with a low wt. $\%$ graphene. The graphene paper usually exhibited a shiny metallic luster on both sides. These papers have been found quite smooth and throughout the whole cross-section, the fracture edges displayed a layered structure comparable to GO paper microstructure. Similar to GO paper, graphene paper can also be prepared using vacuum filtration. These results showed that the chemically modified graphene sheets can be arranged under vacuum filtration-induced directional flow to form a highly ordered macroscopic graphene structure. Figure 20 shows the AFM image of exfoliated graphene flakes. ${ }^{134}$ By adjusting the colloidal suspension volume, the graphene paper thickness was varied from $10 \mathrm{~nm}$ to $10 \mu \mathrm{m}$. However, smooth, uniform and shiny papers could only be produced from agglomerate free and stable graphene colloids. As compared to GO paper, graphene paper displaying outstanding water-resistance properties were not easily redispersed into water by ultrasonication.

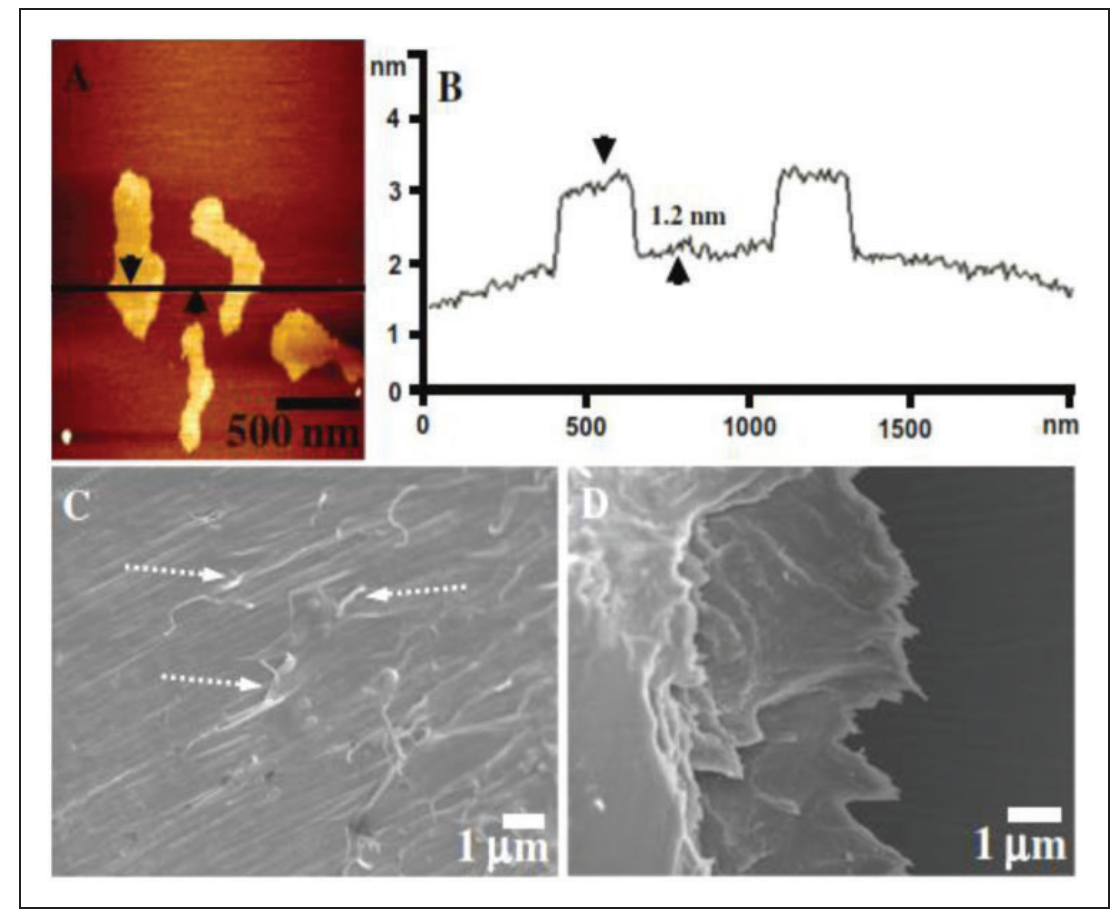

Figure 20. (a) AFM image of the exfoliated graphene flakes, (b) height profile of one of the graphene flakes, (c) FESEM image of graphene/PVC composite thin film at 2 wt. \% concentration and (d) FESEM image of the fractured composite film after mechanical testing. ${ }^{134}$ 
The graphene papers were electrically conductive and mechanically strong with conductivity of $351 \mathrm{Scm}^{-1}, 293.3 \mathrm{MPa}$ tensile strength, and $41.8 \mathrm{GPa}$ young modulus. These robust, flexible freestanding graphene papers may find their use in energy storage devices. ${ }^{135}$ Various properties such as mechanical, thermal and electrical have been reported in order to check the dominancy of graphene. The influence of graphene as a nanofiller material has been studied in many polymeric systems such as polycarbonate (PC), polystyrene (PS), polyaniline (PANI), polyethylene terephthalate (PET), polyurethane (PU), Nafion, and polyvinylidene fluoride (PVDF), in order to produce a graphene-based polymer nanocomposite with high performance for a many potential applications. ${ }^{136}$ In many cases, polymer/graphene composites have been predicted to encompass multifunctional characteristics. Along with mechanical properties, thermal, and electrical conductivity have also been improved. Therefore, graphene has been more appropriate as a filler material because of its excellent mechanical properties. Gómez-Navarro et al. ${ }^{137}$ described that the elastic modulus of graphene was $250 \mathrm{GPa}$ (36 Million psi) versus GO at 207.6 $\pm 23.4 \mathrm{GPa}$ (30 Million \pm 3.4 Million psi). Figure 21 shows the mechanical properties of $\mathrm{PU} /$ graphene composite film versus graphene content. ${ }^{138}$

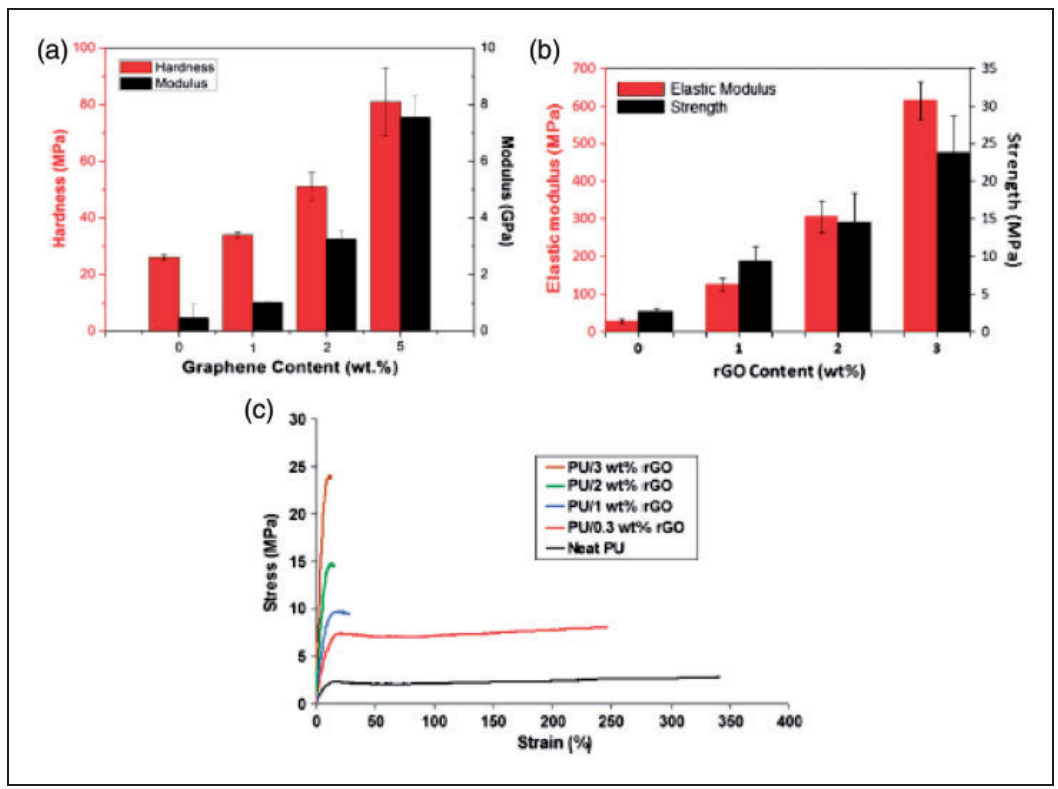

Figure 2I. PU/rGO composites (a) hardness via nanoindentation versus graphene content, (b) elastic Modulus and Tensile Strength for rGO composites versus rGO content versus, (c) typical tensile stress versus strain curves for PU/rGO composites containing different graphene content. ${ }^{138}$ 
Graphene has higher thermal and electrical conductivity than GO. Due to graphene's different surface chemistry its effectiveness to enhance the mechanical properties of a polymer/graphene composite was totally different from the GO composites. This resulted in different interfacial interaction between matrices and filler, different polymer matrix crystallinity and different filler dispersibility states. High thermal stability was another excellent property of graphene paper as compared to $\mathrm{GO}$ paper. Below $200^{\circ} \mathrm{C}$, the mass loss was accredited to removing adsorbed water. At $200-500^{\circ} \mathrm{C}$, a slight mass loss appeared probably due to the decomposition of some remaining oxygen containing functional groups. The above results show that the reduced graphene paper was more thermally stable than GO paper. As compared to GO, exfoliated graphite has also been employed as filler for the layered composites reported by Kausar et al. ${ }^{139}$ Graphite was functionalized by oxidizing natural graphite. The oxidation widened the gap between graphite layers facilitating the pyrrole monomers to polymerize into the graphite galleries. Afterward layer by layer polymerization was used to form multilayered composites.

Wang et al. ${ }^{140}$ combined the advantages of conducting polymer (large capacitance) and graphene paper (flexibility and high conductivity) and synthesized graphene-conducting PANI composite paper as a freestanding flexible electrode. The conducting polymer, PANI is commonly known to enhance the electrochemical capacitance of carbon based materials. For example, PANI modified mesoporous carbon and carbon monolith displayed considerably enhanced capacitance. Hence, adding PANI was predicted to maximize the unique properties of freestanding graphene paper as a flexible electrode for supercapacitors. For this purpose, they prepared polyaniline/ graphene composite paper (GPCP) through in-situ anodic electropolymerization (AEP) on graphene paper. This composite paper had excellent conductivity, flexibility and electrochemical activity. Moreover, it showed excellent volumetric capacitance of $135 \mathrm{~F} \mathrm{~cm}^{-3}$ and gravimetric capacitance of $233 \mathrm{Fg}^{-1}$, outperforming several other presently accessible carbon-based freestanding electrodes. ${ }^{140}$ In GPCP, polyaniline is distributed two ways:

- a two dimensional PANI distribution on the graphene sheet surface as a building unit for GPCP paper

- a three dimensional PANI distribution on the interior and exterior parts of the GPCP paper.

The graphene composite paper's mechanical properties are $(12.6 \mathrm{MPa}$ tensile strength at 0.11 strain) which is higher than graphene paper (8.8 MPa tensile strength at 0.08 strain). The higher tensile strength of graphene polymer composite paper was due to improved mechanical interconnection among the polymer/graphene layers. The higher tensile strength determines the mechanical durability of polymer/graphene composite paper for flexible supercapacitors as binderless and current collector-free electrodes. 
However, the tensile strength of graphene paper and GPCP paper was lower than graphene oxide papers. This was possibly due to different graphene properties and structure and different infiltration conditions for paper formation. ${ }^{141}$ The GPCP and G-paper exhibited porous characteristics with a specific surface area of 39 and $94 \mathrm{~m}^{2} \mathrm{~g}^{-1}$, respectively. By reducing the porosity, the G-paper strength further improved which enhanced the GPCP tensile property. However, this may reduce the electrochemical accessible areas. Therefore, it was necessary to maintain a balance between tensile strength and porosity when fabricating advanced GPCP flexible electrodes.

\section{Conclusions and future potential}

Flexible energy storage devices formed from graphene oxide, graphene and polymer/GO paper-like materials have various novel applications in moveable electronic devices including electronic paper, roll-up displays, wearable systems for special computing, multimedia or medical devices, and stretchable interconnected circuits. Flexible supercapacitors made from paper-like materials have outstanding properties such as long cycle life, good operational safety, moderate energy density, and large power density. Therefore, these are highly anticipated as current energy storage devices. A freestanding binder-free electrode, with large capacitance and promising mechanical strength, is a potential source for flexible supercapacitors, although conducting polymer and transition metal oxide have been extensively considered as supercapacitor electrode materials. Only carbon based paper-like materials exhibit promising flexibility and hence been favored as self-supporting soft electrodes. Films, papers, and clothes made from CNT/ fibers have been recognized as appropriate freestanding electrodes. However, these electrodes always have high capacitance due to the less active surfaces of carbon-based materials. Consequently, including a second phase, which is electrochemically active into a freestanding electrode containing carbon based materials, can improve the electrode capacitance. Graphene oxide obtained from exfoliated graphite provides a practical way towards flexible, low-cost, bulk production, ease of deposition and outstanding optoelectronics properties of paper-like materials. The unique features of GO are solubility in various solvents that allows incorporating GO into composites to enhance its properties. Various techniques have been used to achieve graphene oxide, graphene, and $\mathrm{GO} /$ graphene-based composite papers that allow fabricating flexible energy storage devices, transparent conductors, sensors, medical devices, memory devices, and photovoltaics. Although further work is required to complete the advanced graphene-based electrodes, they have the advantage of high-through-put and low-cost deposition for novel applications. To understand the processing, structure and fundamental 
properties of $\mathrm{GO} /$ graphene-based papers, more development has to be made in order to enhance and explore the highly versatile properties of composites. GO offer an exciting platform towards physics, engineering, materials science, and chemistry of an exceptional 2D system. In addition to these, it also provides a way to study carbon-based thin paper technology. Continuous research from all disciplines should further enhance the potential of $\mathrm{GO} /$ graphene paper-like material as an electrode in energy storage devices.

\section{Declaration of Conflicting Interests}

The author(s) declared no potential conflicts of interest with respect to the research, authorship, and/or publication of this article.

\section{Funding}

The author(s) received no financial support for the research, authorship, and/or publication of this article.

\section{References}

1. Boehm HP, Setton R and Stumpp E. Nomenclature and terminology of graphite intercalation compounds. Pure Appl Chem 1994; 66: 1893-1901.

2. Sattar R, Kausar A and Siddiq M. Advances in thermoplastic polyurethane composites reinforced with carbon nanotubes and carbon nanofibers: A review. J Plast Film Sheet 2015; 31: 186-224.

3. Stankovich S, Dikin DA, Piner RD, et al. Synthesis of graphene-based nanosheets via chemical reduction of exfoliated graphite oxide. Carbon 2007; 45: $1558-1565$.

4. Watcharotone S, Dikin DA, Stankovich S, et al. Graphene-silica composite thin films as transparent conductors. Nano Lett 2007; 7: 1888-1892.

5. Eda G and Chhowalla M. Chemically derived graphene oxide: Towards large-area thin-film electronics and optoelectronics. Adv Mater 2010; 22: 2392-2415.

6. Cao Q and Rogers J. Ultrathin films of single-walled carbon nanotubes for electronics and sensors: A review of fundamental and applied aspects. Adv Mater 2008; 20: 29-53.

7. Brodie BC. On the atomic weight of graphite. Philos Trans R Soc London 1859; 149: 249-259.

8. Hummers Jr WS and Offeman RE. Preparation of graphitic oxide. J Am Chem Soc 1958; 80: 1339-1339.

9. Boehm HP, Clauss A, Fischer G, et al. Surface properties of extremely thin graphite lamellae. In: Proceedings of the fifth conference on carbon, Pennsylvania State University, Pennsylvania, USA, 1962, pp. 73-80.

10. Shelton JC, Patil HR and Blakely JM. Equilibrium segregation of carbon to a nickel (III) surface: A surface phase transition. Surf Sci 1974; 43: 493-520.

11. IUPAC. Compendium of chemical terminology. McNaught AD and Wilkinson A (eds), 2nd ed. Oxford:Blackwell Scientific, 1997. 
12. Novoselov KS, Geim AK, Morozov SV, et al. Electric field effect in atomically thin carbon films. Science 2004; 306: 666-669.

13. Rao CNR, Biswas K, Subrahmanyam KS, et al. Graphene, the new nanocarbon. J Mater Chem 2009; 19: 2457-2469.

14. Rao CNR, Sood AK, Subrahmanyam KS, et al. Graphene: The new twodimensional nanomaterial. Angew Chem Int Ed 2009; 48: 7752-7777.

15. Liu Z, Liu J, Cui L, et al. Preparation of graphene/polymer composites by direct exfoliation of graphite in functionalised block copolymer matrix. Carbon 2013; 51: 148-155.

16. Chen D, Tang L and Li J. Graphene-based materials in electrochemistry. Chem Soc Rev 2010; 39: 3157-3180.

17. Wallace PR. The band theory of graphite. Phys Rev 1947; 71: 622-634.

18. Novoselov KS, Jiang Z, Zhang Y, et al. Room-temperature quantum Hall effect in graphene. Science 2007; 315: 1379-1379.

19. Lu X, Huang H, Nemchuk N, et al. Patterning of highly oriented pyrolytic graphite by oxygen plasma etching. Appl Phys Lett 1999; 75: 193-195.

20. Song L, Ci L, Gao W, et al. Transfer printing of graphene using gold film. ACS Nano 2009; 3: 1353-1356.

21. Kong J, Cassell AM and Dai H. Chemical vapor deposition of methane for singlewalled carbon nanotubes. Chem Phys Lett 1998; 292: 567-574.

22. Reina A, Jia X, Ho J, et al. Large area, few-layer graphene films on arbitrary substrates by chemical vapor deposition. Nano Lett 2008; 9: 30-35.

23. Hernandez Y, Nicolosi V, Lotya M, et al. High-yield production of graphene by liquid-phase exfoliation of graphite. Nat Nanotechnol 2008; 3: 563-568.

24. Vallés C, Drummond C, Saadaoui H, et al. Solutions of negatively charged graphene sheets and ribbons. J Am Chem Soc 2008; 130: 15802-15804.

25. Li X, Zhang G, Bai X, et al. Highly conducting graphene sheets and LangmuirBlodgett films. Nat Nanotechnol 2008; 3: 538-542.

26. Hao R, Qian W, Zhang L, et al. Aqueous dispersions of TCNQ-anion-stabilized graphene sheets. Chem Commun 2008; 48: 6576-6578.

27. Park S and Ruoff RS. Chemical methods for the production of graphenes. Nat Nanotechnol 2009; 4: 217-224.

28. Novoselov KS, Geim AK, Morozov SV, et al. Electric field effect in atomically thin carbon films. Science 2004; 306: 666-969.

29. Jang JY, Kim MS, Jeong HM, et al. Graphite oxide/poly(methyl methacrylate) nanocomposites prepared by a novel method utilizing macroazoinitiator. Compos Sci Technol 2009; 69: 186-191.

30. Buchsteiner A, Lerf A and Pieper J. Water dynamics in graphite oxide investigated with neutron scattering. J Phys Chem B 2006; 110: 22328-22338.

31. $\mathrm{Hu} \mathrm{H}$, Wang $\mathrm{X}$, Wang $\mathrm{J}$, et al. Preparation and properties of graphene nanosheets - polystyrene nanocomposites via in-situ emulsion polymerization. Chem Phys Lett 2010; 484: 247-253.

32. Li D, Müller MB, Gilje S, et al. Processable aqueous dispersions of graphene nanosheets. Nat Nanotechnol 2008; 3: 101-105. 
33. de Moraes ACM, Andrade PF, de Faria AF, et al. Fabrication of transparent and ultraviolet shielding composite films based on graphene oxide and cellulose acetate. Carbohyd Polym 2015; 123: 217-227.

34. Paredes JI, Villar-Rodil S, Martinez-Alonso A, et al. Graphene oxide dispersions in organic solvents. Langmuir 2008; 24: 10560-10564.

35. Viinikanoja A, Wang Z, Kauppila J, et al. Electrochemical reduction of graphene oxide and its in situ spectroelectrochemical characterization. Phys Chem Chem Phys 2012; 14: 14003-14009.

36. Schafheutl C. LXXXVI. On the combinations of carbon with silicon and iron, and other metals, forming the different species of cast iron, steel, and malleable iron. Philos Mag 1840; 16: 570-590.

37. Brodie BC. On the atomic weight of graphite. Philos Trans R Soc London 1859; 149: $249-259$.

38. Staudenmaier L. Verfahren zur Darstellung der Graphitsäure. Ber Dtsch Chem Ges 1898; 31: 1481-1487.

39. Hummers Jr WS and Offeman RE. Preparation of graphitic oxide. J Am Chem Soc 1958; 80: 1339-1339.

40. Zhang N, Wang LY, Liu H, et al. Nitric acid oxidation on carbon dispersion and suspension stability. Surf Interface Anal 2008; 40: 1190-1194.

41. Simon A, Dronskowski R, Krebs B, et al. The crystal structure of Mn2O7. Angew Chem Int Ed 1987; 26: 139-140.

42. Wissler M. Graphite and carbon powders for electrochemical applications. J Power Source 2006; 156: 142-150.

43. Zhu Y, Murali S, Cai W, et al. Graphene and graphene oxide: Synthesis, properties, and applications. Adv Mater 2010; 22: 3906-3924.

44. Marcano DC, Kosynkin DV, Berlin JM, et al. Improved synthesis of graphene oxide. ACS Nano 2010; 4: 4806-4814.

45. Hofmann U and Holst R. Über die Säurenatur und die Methylierung von Graphitoxyd. Ber Dtsch Chem Ges B 1939; 72: 754-771.

46. Ruess G. Über das graphitoxyhydroxyd (graphitoxyd). Monatsh Chem 1946; 76: 381-417.

47. Mermoux M, Chabre Y and Rousseau A. FTIR and ${ }^{13} \mathrm{C}$ NMR study of graphite oxide. Carbon 1991; 29: 469-474.

48. Dubois M, Giraudet J, Guerin K, et al. EPR and solid-state NMR studies of poly (dicarbon monofluoride)(C2F) $)_{\mathrm{n}}$. J Phys Chem B 2006; 110: 11800-11808.

49. Scholz W and Boehm HP. Graphite oxide.6. Structure of graphite oxide. Z Anorg Allg Chem 1969; 369: 327-340.

50. Nakajima T, Mabuchi A and Hagiwara R. A new structure model of graphite oxide. Carbon 1988; 26: 357-361.

51. Lerf-Klinowski A, He H, Forster M, et al. Structure of graphite oxide revisited. J Phys Chem B 1998; 102: 4477-4482.

52. Szabó T, Berkesi O, Forgó P, et al. Evolution of surface functional groups in a series of progressively oxidized graphite oxides. Chem Mater 2006; 18: 2740-2749. 
53. He H, Riedl T, Lerf A, et al. Solid-state NMR studies of the structure of graphite oxide. J Phys Chem 1996; 100: 19954-19958.

54. Cai W, Piner RD, Stadermann FJ, et al. Synthesis and solid-state NMR structural characterization of ${ }^{13} \mathrm{C}$-labeled graphite oxide. Science 2008; 321: 1815-1817.

55. Casabianca LB, Shaibat MA, Cai WW, et al. NMR-based structural modeling of graphite oxide using multidimensional 13C solid-state NMR and ab initio chemical shift calculations. J Am Chem Soc 2010; 132: 5672-5676.

56. Becerril HA, Mao J, Liu Z, et al. Evaluation of solution-processed reduced graphene oxide films as transparent conductors. ACS Nano 2008; 2: 463-470.

57. Stankovich S, Piner RD, Chen X, et al. Stable aqueous dispersions of graphitic nanoplatelets via the reduction of exfoliated graphite oxide in the presence of poly (sodium 4-styrenesulfonate). J Mater Chem 2006; 16: 155-158.

58. Cote LJ, Kim J, Tung VC, et al. Graphene oxide as surfactant sheets. Pure Appl Chem 2010; 83: 95-110.

59. Skoog DA, Holler FJ and Nieman TA. Principles of instrumental analysis. Philadelphia: Hartcourt Brace \& Company, 1998, Chap 13, pp. 839-849.

60. Riddick JA, Bunger WB and Sakano TK. Organic solvents: Physical properties and methods of Purification, 4th ed. New York: John Wiley \& Sons, 1986, pp.135-147.

61. Akhavan $\mathrm{O}$ and Ghaderi E. Toxicity of graphene and graphene oxide nanowalls against bacteria. ACS Nano 2010; 4: 5731-5736.

62. Hu W, Peng C, Lv M, et al. Protein corona-mediated mitigation of cytotoxicity of graphene oxide. ACS Nano 2011; 5: 3693-3700.

63. Wang K, Ruan J, Song H, et al. Biocompatibility of graphene oxide. Nanoscale Res Lett 2011; 6: 1-8.

64. Zhang Y, Ali SF, Dervishi E, et al. Cytotoxicity effects of graphene and singlewall carbon nanotubes in neural phaeochromocytoma-derived PC12 cells. ACS Nano 2010; 4: 3181-3186.

65. Ruiz ON, Fernando KS, Wang B, et al. Graphene oxide: A nonspecific enhancer of cellular growth. ACS Nano 2011; 5: 8100-8107.

66. Dikin DA, Stankovich S, Zimney EJ, et al. Preparation and characterization of graphene oxide paper. Nature 2007; 448: 457-460.

67. Lerf A, Buchsteiner A, Pieper J, et al. Hydration behavior and dynamics of water molecules in graphite oxide. J Phys Chem Sol 2006; 67: 1106-1110.

68. Medhekar NV, Ramasubramaniam A, Ruoff RS, et al. Hydrogen bond networks in graphene oxide composite paper: Structure and mechanical properties. ACS Nano 2010; 4: 2300-2306.

69. Buchsteiner A, Lerf A and Pieper J. Water dynamics in graphite oxide investigated with neutron scattering. J Phys Chem B 2006; 110: 22328-22338.

70. Hofmann $U$ and Frenzel A. The reduction of graphite oxide with hydrogen sulphide. Kolloid-Zeitschrift 1934; 68: 149-151.

71. Chen Y, Zhang X, Yu P, et al. Stable dispersions of graphene and highly conducting graphene films: A new approach to creating colloids of graphene monolayers. Chem Commun 2009; 30: 4527-4529. 
72. Fan X, Peng W, Li Y, et al. Deoxygenation of exfoliated graphite oxide under alkaline conditions: A green route to graphene preparation. Adv Mater 2008; 20 : 4490-4493.

73. Shin HJ, Kim KK, Benayad A, et al. H. Efficient reduction of graphite oxide by sodium borohydride and its effect on electrical conductance. Adv Funct Mater 2009; 19: 1987-1992.

74. Schniepp HC, Li JL, McAllister MJ, et al. Functionalized single graphene sheets derived from splitting graphite oxide. J Phys Chem B 2006; 110: 8535-8539.

75. Zhou M, Wang Y, Zha Y, et al. Controlled synthesis of large-area and patterned electrochemically reduced graphene oxide films. Chem-A Eur J 2009; 15: 6116-6120.

76. Mohanty N and Berry V. Graphene-based single-bacterium resolution biodevice and DNA transistor: Interfacing graphene derivatives with nanoscale and microscale biocomponents. Nano Lett 2008; 8: 4469-4476.

77. Yang X, Zhang X, Liu Z, et al. High-efficiency loading and controlled release of doxorubicin hydrochloride on graphene oxide. J Phys Chem C 2008; 112: 17554-17558.

78. Dai L, Lu J, Matthews B, et al. Doping of conducting polymers by sulfonated fullerene derivatives and dendrimers. J Phys Chem B 1998; 102: 4049-4053.

79. Chapartegui M, Barcena J, Irastorza X, et al. Analysis of the conditions to manufacture a MWCNT buckypaper/benzoxazine nanocomposite. Compos Sci Technol 2012; 72: 489--497.

80. Chen CM, Yang QH, Yang YG, et al. Self-assembled free standing graphite oxide membrane. Adv Mater 2009; 21: 3007-3011.

81. Stankovich S, Piner R, Nguyen ST, et al. Synthesis and exfoliation of isocyanatetreated graphene oxide nanoplatelets. Carbon 2006; 44: 3342-3347.

82. Huang ZD, Zhang B, Oh SW, et al. Self-assembled reduced graphene oxide/ carbon nanotube thin film as electrodes for supercapacitors. J Mater Chem 2012; 22: 3591-3599.

83. Yousefi N, Gudarzi MM, Zheng QB, et al. Self-alignment and high electrical conductivity of ultralarge grapheneoxide-polyurethane nanocomposites. J Mater Chem 2012; 22: 12709-1217.

84. Li D, Muller MB, Gilje S, et al. Processable aqueous dispersions of graphene nanosheets. Nat Nanotechnol 2008; 3: 101-105.

85. Huang ZD, Zhang B, Liang R, et al. Effects of reduction process and carbon nanotube content on supercapacitive performance of flexible graphene oxide papers. Carbon 2012; 50: 4239-4251.

86. Su Y, Wei H, Gao R, et al. Exceptional negative thermal expansion and viscoelastic properties of graphene oxide paper. Carbon 2012; 50: 2804-2809.

87. Park S, Lee KS, Bozoklu G, et al. Graphene oxide papers modified by divalent ions-enhancing mechanical properties via chemical cross-linking. ACS Nano 2008; 2: $572-578$.

88. Tian Y, Cao Y, Wang Y, et al. Realizing ultrahigh modulus and high strength of macroscopic graphene oxide papers through crosslinking of mussel-inspired polymers. Adv Mater 2013; 25: 2980-2983. 
89. Gao Y, Liu LQ, Zu SZ, et al. The effect of interlayer adhesion on the mechanical behaviors of macroscopic graphene oxide papers. ACS Nano 2011; 5: 2134-2141.

90. Compton OC, Cranford SW, Putz KW, et al. Tuning the mechanical properties of graphene oxide paper and its associated polymer nanocomposites by controlling cooperative intersheets hydrogen bonding. ACS Nano 2012; 6: 2008-2019.

91. Cano M, Khan U, Sainsbury T, et al. Improving the mechanical properties of graphene oxide based materials by covalent attachment of polymer chains. Carbon 2013; 52: 363-371.

92. Lee W, Lee JU, Jung BM, et al. Simultaneous enhancement of mechanical, electrical and thermal properties of graphene oxide paper by embedding dopamine. Carbon 2013; 65: 296-304.

93. Jin E, He J, Sheng K, et al. Electronirradiation-induced reinforcement of reduced graphene oxide papers. Acta Mater 2013; 61: 6466-6473.

94. Cerveny S, Barroso-Bujans F, Alegria A, et al. Dynamics of water intercalation in graphite oxide. J Phys Chem C 2010; 114: 2604-2612.

95. Lin X, Shen X, Zheng Q, et al. Fabrication of highly-aligned, conductive, and strong graphene papers using ultralarge graphene oxide sheets. ACS Nano 2012; 6: 10708-10719.

96. Mattevi C, Eda G, Agnoli S, et al. Evolution of electrical, chemical, and structural properties of transparent and conducting chemically derived graphene thin films. Adv Funct Mater 2009; 19: 2577-2583.

97. Ou E, Zhang X, Chen Z, et al. Macroscopic, free-standing Ag-reduced, graphene oxide janus films prepared by evaporation-induced self-assembly. Chem-A Eur J 2011; 17: 8789-8793.

98. Robinson JT, Zalalutdinov M, Baldwin JW, et al. Wafer-scale reduced graphene oxide films for nanomechanical devices. Nano Lett 2008; 8: 3441-3445.

99. Schniepp HC, Li JL, McAllister MJ, et al. Functionalized single graphene sheets derived from splitting graphite oxide. J Phys Chem B 2006; 110: 8535-8539.

100. Cote LJ, Kim F and Huang J. Langmuir-Blodgett assembly of graphite oxide single layers. J Am Chem Soc 2008; 131: 1043-1049.

101. Gilje S, Han S, Wang M, et al. A chemical route to graphene for device applications. Nano Lett 2007; 7: 3394-3398.

102. Wang X, Zhi L and Müllen K. Transparent, conductive graphene electrodes for dye-sensitized solar cells. Nano Lett 2008; 8: 323-327.

103. Gengler RY, Veligura A, Enotiadis A, et al. Large-yield preparation of highelectronic-quality graphene by a Langmuir-Schaefer approach. Small 2010; 6: $35-39$.

104. Eda G, Fanchini G and Chhowalla M. Large-area ultrathin films of reduced graphene oxide as a transparent and flexible electronic material. Nat Nanotechnol 2008; 3: 270-274.

105. Becerril HA, Mao J, Liu Z, et al. Evaluation of solution-processed reduced graphene oxide films as transparent conductors. ACS Nano 2008; 2: 463-470. 
106. Park S, An J, Suk JW, et al. Graphene-based actuators. Small 2010; 6: 210-212.

107. Hennrich F, Lebedkin S, Malik S, et al. Preparation, characterization and applications of free-standing single walled carbon nanotube thin films. Phys Chem Chem Phys 2002; 4: 2273-2277.

108. Robinson JT, Zalalutdinov M, Baldwin JW, et al. Wafer-scale reduced graphene oxide films for nanomechanical devices. Nano Lett 2008; 8: 3441-3445.

109. Yamaguchi H, Eda G, Mattevi C, et al. Highly uniform $300 \mathrm{~mm}$ wafer-scale deposition of single and multilayered chemically derived graphene thin films. ACS Nano 2010; 4: 524-528.

110. Li X, Zhang G, Bai X, et al. Highly conducting graphene sheets and LangmuirBlodgett films. Nat Nanotechnol 2008; 3: 538-542.

111. Loh KP, Bao Q, Eda G, et al. Graphene oxide as a chemically tunable platform for optical applications. Nat Chem 2010; 2: 1015-1024.

112. Xie S, Zhang B, Wang C, et al. Building up graphene-based conductive polymer composite thin films using reduced graphene oxide prepared by $\gamma$-ray irradiation. Sci World J 2013; 2013: 1-7.

113. Wu Z, Chen Z, Du X, et al. Transparent, conductive carbon nanotube films. Science 2004; 305: 1273-1276.

114. Fan J, Shi Z, Ge Y, et al. Gum arabic assisted exfoliation and fabrication of Ag-graphene-based hybrids. J Mater Chem 2012; 22: 13764-13772.

115. McConnell MD, Kraeutler MJ, Yang S, et al. Patchy and multiregion janus particles with tunable optical properties. Nano Lett 2010; 10: 603-609.

116. Pitkethly MJ. Nanomaterials - the driving force. Mater Today 2004; 7: 20-29.

117. Kubacka A, Serrano C, Ferrer M, et al. High-performance dual-action polymer- $\mathrm{TiO}_{2}$ nanocomposite films via melting processing. Nano Lett 2007; 7: 2529-2534.

118. Wang DW, Li F, Zhao J, et al. Fabrication of graphene/polyaniline composite paper via in situ anodic electropolymerization for high-performance flexible electrode. ACS Nano 2009; 3: 1745-1752.

119. MacDiarmid AG. "Synthetic metals": A novel role for organic polymers (Nobel lecture). Angew Chem Int Ed 2001; 40: 2581-2590.

120. Szabó T, Berkesi O, Forgó P, et al. Evolution of surface functional groups in a series of progressively oxidized graphite oxides. Chem Mater 2006; 18: 2740-2749.

121. Zhang F, Kang ET, Neoh KG, et al. Reactive coupling of poly (ethylene glycol) on electroactive polyaniline films for reduction in protein adsorption and platelet adhesion. Biomaterials 2002; 23: 787-795.

122. Stoller MD, Park S, Zhu Y, et al. Graphene-based ultracapacitors. Nano Lett 2008; 8: 3498-3502.

123. Lerf A, Buchsteiner A, Pieper J, et al. Hydration behavior and dynamics of water molecules in graphite oxide. J Phys Chem Sol 2006; 67: 1106-1110.

124. Buchsteiner A, Lerf A and Pieper J. Water dynamics in graphite oxide investigated with neutron scattering. J Phys Chem B 2006; 110: 22328-22338. 
125. Liang J, Huang Y, Zhang L, et al. Molecula-level dispersion of graphene into poly (vinyl alcohol) and effective reinforcement of their nanocomposites. Adv Funct Mater 2009; 19: 2297-2302.

126. Lee H, Dellatore SM, Miller WM, et al. Mussel-inspired surface chemistry for multifunctional coatings. Science 2007; 318: 426-430.

127. Ryu S, Lee Y, Hwang JW, et al. High strength carbon nanotube fibers fabricated by infiltration and curing of mussel-inspired catecholamine polymer. Adv Mater 2011; 23: 1971-1975.

128. Novoselov KS, Jiang Z, Zhang Y, et al. Room-temperature quantum Hall effect in graphene. Science 2007; 315: 1379-1379.

129. Stoller MD, Park S, Zhu Y, et al. Graphene-based ultracapacitors. Nano Lett 2008; 8: 3498-3502.

130. Li D, Muller MB, Gilje S, et al. Processable aqueous dispersions of graphene nanosheets. Nat Nanotechnol 2008; 3: 101-105.

131. Ramanathan T, Abdala AA, Stankovich S, et al. Functionalized graphene sheets for polymer nanocomposites. Nat Nanotechnol 2008; 3: 327-331.

132. Li D and Kaner RB. Graphene based materials. Science 2008; 320: 1170-1171.

133. Shah R, Kausar A and Muhammad B. Exploration of polythiophene/graphene, Poly(methyl methacrylate)/graphene and PTh-co-PMMA/graphene hybrids obtained via in-situ technique. J Plast Film Sheet 2015; 31: 144-157.

134. Vadukumpully S, Paul J, Mahanta N, et al. Flexible conductive graphene/poly (vinyl chloride) composite thin films with high mechanical strength and thermal stability. Carbon 2011; 49: 198-205.

135. Chen H, Müller MB, Gilmore KJ, et al. Mechanically strong, electrically conductive, and biocompatible graphene paper. Adv Mater 2008; 20: 3557-3561.

136. Kuilla T, Bhadra S, Yao D, et al. Recent advances in graphene based polymer composites. Prog Polym Sci 2010; 35: 1350-1375.

137. Gómez-Navarro C, Weitz RT, Bittner AM, et al. Electronic transport properties of individual chemically reduced graphene oxide sheets. Nano Lett 2007; 7: 3499-3503.

138. Yousefi N, Gudarzi MM, Zheng Q, et al. Highly aligned, ultralarge-size reduced graphene oxide/polyurethane nanocomposites: Mechanical properties and moisture permeability. Compos Part A Appl Sci Manufact 2013; 49: 42-50.

139. Naz A, Kausar A and Siddiq M. Preparation and properties of exploration of high performance multi-layered polypyrrole/polyethylene glycol/poly(styreneco-maleic anhydride)cumene terminated/4,4'methylenedianiline/graphite-based nanocomposites. J Plast Film Sheet 2014; 30: 388-411.

140. Wang DW, Li F, Zhao J, et al. Fabrication of graphene/polyaniline composite paper via in situ anodic electropolymerization for high-performance flexible electrode. ACS Nano 2009; 3: 1745-1752.

141. Xu YX, Bai H, Lu GW, et al. Flexible graphene films via the filtration of watersoluble noncovalent functionalized graphene sheets. J Am Chem Soc 2008; 130: $5856-5857$. 


\section{Biographies}

Zaheen U Khan obtained his MPhil from National Centre for Physics, Quaid-i-Azam University, Islamabad, Pakistan. At present, he is involved in fabricating novel polymer intercalated buckypaper composites.

Ayesha Kausar is currently serving National Centre for Physics, Quaid-i-Azam University, Islamabad, Pakistan. She obtained her PhD from Quaidi-Azam University, Islamabad/KAIST (Korea Advanced Institute of Science \& Technology), Graduate School of EEWS, Daejeon, Republic of Korea. Her research interests include synthesis, characterization and structure-property relationship of new types of polymeric materials; synthesis of nanomaterials including organic-inorganic nanocomposites/hybrid materials; polymeric blends via incorporation of nanoparticles into thin polymer films; exploration of practical and potential prospects of the novel synthesized materials (new polymers and nanomaterials) counting morphological, mechanical, thermal, electrical, conducting, etc.; flame retardant materials; proton conducting fuel cell membranes; nanocomposites for polymer Li-ion battery electrodes; composites based on electrospun nanofibers; production of various types of polymeric nanoparticles and their composites for solar cells; polymer/carbon nanotube/nanoparticle composites for water treatment; potential of polymer/ graphite nanocomposites; polymer/graphene hybrids; polymer/fullerene nanomaterials; fabrication of epoxy-based nanocomposite for various applications; radar absorbing materials; aerospace relevance, and so on.

Hidayat Ullah is an Assistant Professor in Institute of Chemical Sciences, Gomal University, Dera Ismail Khan, Pakistan. His current research interest is graphene-based materials.

Amin Badshah is currently a Professor and head of Inorganic/Analytical chemistry department in Quaid-i-Azam University Campus, Islamabad, Pakistan. His research interests include inorganic materials, medicinal chemistry, synthesis, crystal structure, etc.

Wasid U Khan is a PhD scholar in Analytical Chemistry Department, Institute of Chemistry, São Paulo State University, 14801-970 Araraquara$\mathrm{SP}$, Brazil. He is exploring functional graphene-based materials. 